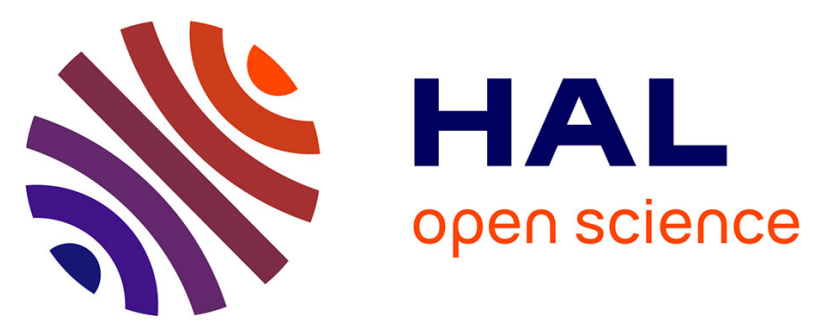

\title{
Noise and ergodic properties of Brownian motion in an optical tweezer: Looking at regime crossovers in an Ornstein-Uhlenbeck process
}

Rémi Goerlich, Minghao Li, Samuel Albert, Giovanni Manfredi, Paul-Antoine Hervieux, Cyriaque Genet

\section{To cite this version:}

Rémi Goerlich, Minghao Li, Samuel Albert, Giovanni Manfredi, Paul-Antoine Hervieux, et al.. Noise and ergodic properties of Brownian motion in an optical tweezer: Looking at regime crossovers in an Ornstein-Uhlenbeck process. Physical Review E , 2021, 103 (3), 10.1103/PhysRevE.103.032132 . hal-03417915

\section{HAL Id: hal-03417915 \\ https://hal.science/hal-03417915}

Submitted on 17 Nov 2021

HAL is a multi-disciplinary open access archive for the deposit and dissemination of scientific research documents, whether they are published or not. The documents may come from teaching and research institutions in France or abroad, or from public or private research centers.
L'archive ouverte pluridisciplinaire HAL, est destinée au dépôt et à la diffusion de documents scientifiques de niveau recherche, publiés ou non, émanant des établissements d'enseignement et de recherche français ou étrangers, des laboratoires publics ou privés. 


\title{
Noise and ergodic properties of Brownian motion in an optical tweezer: Looking at regime crossovers in an Ornstein-Uhlenbeck process
}

\author{
Rémi Goerlich $\odot,{ }^{1,2, *}$ Minghao Li $\odot,{ }^{2, *}$ Samuel Albert $\odot,{ }^{2}$ Giovanni Manfredi $\odot,{ }^{1, \dagger}$ \\ Paul-Antoine Hervieux, ${ }^{1, \$}$ and Cyriaque Genet $\odot^{2, \S}$ \\ ${ }^{1}$ Université de Strasbourg, CNRS, Institut de Physique et Chimie des Matériaux de Strasbourg, UMR 7504, F-67000 Strasbourg, France \\ ${ }^{2}$ Université de Strasbourg, CNRS, Institut de Science et d'Ingénierie Supramoléculaires, UMR 7006, F-67000 Strasbourg, France
}

(Received 29 September 2020; revised 5 January 2021; accepted 15 February 2021; published 19 March 2021)

\begin{abstract}
We characterize throughout the spectral range of an optical trap the nature of the noise that drives the Brownian motion of an overdamped trapped single microsphere and its ergodicity, comparing experimental, analytical, and simulated data. We carefully analyze noise and ergodic properties (i) using the Allan variance for characterizing the noise and (ii) exploiting a test of ergodicity tailored for experiments done over finite times. We derive these two estimators in the Ornstein-Uhlenbeck low-frequency trapped-diffusion regime and study analytically their evolution toward the high-frequency Wiener-like free-diffusion regime, in very good agreement with simulated and experimental results. This study is performed comprehensively from the free-diffusion to the trapped-diffusion regimes. It also carefully looks at the specific signatures of the estimators at the crossover between the two regimes. This analysis is important to conduct when exploiting optical traps in a metrology context.
\end{abstract}

DOI: 10.1103/PhysRevE.103.032132

\section{INTRODUCTION}

The sensitivity of optically trapped Brownian particles, combined with long integration times available, makes optical traps outstanding metrological systems. They have therefore been involved in many weak force experiments and have been recognized as excellent systems for implementing and simulating many results and protocols that have been brought forward recently in the field of optomechanics and nonequilibrium statistical physics [1-4].

The high levels of resolution necessary for such experiments are usually reached by setting up large statistical ensembles. However, this capacity relies on strong assumptions regarding the nature and the stability of the dynamics. In weak force experiments, the resolution is determined by the measurement bandwidth and can be improved when single motional trajectories are acquired over integration times as long as possible [5-10]. But this demands experiments thermally limited throughout the entire measurement time. It is therefore crucial to ensure, both in time and frequency domains, the thermal and stationary character of the noise at play. In precision experiments developed in the context of stochastic thermodynamics, ensembles of synchronized trajectories are built from the repetition of a large number of cycles associated with the nonequilibrium protocol under study [11-14]. But the ergodic hypothesis is necessary here to go from one single trajectory on which a series of $N$ cycles has

\footnotetext{
${ }^{*}$ These authors contributed equally.

† giovanni.manfredi@ipcms.unistra.fr

†hervieux@ipcms.unistra.fr

§genet@unistra.fr
}

been operated to an ensemble of $N$ one-cycle trajectories that represent the time evolution of ensemble-average stochastic quantities. For such experiments, therefore, one must assess, besides the nature and stationarity of the noise, the ergodicity of the whole process, i.e., verifying the equivalence between time and ensemble averages.

Considering the importance of these issues in a metrology context, the aim of this paper is to measure the noise and ergodic properties at play in an optical trap. To do so, we set up two estimators: one capable of characterizing the motional noise using the Allan variance and one assessing the ergodicity, exploiting an ergodicity breaking estimator proposed recently [15-17].

An optical trap physically implements an OrnsteinUhlenbeck process through the harmonic trapping potential and, interestingly, gives access to different diffusing dynamics for the trapped Brownian object, ranging from confined motion in the long timescales to free Brownian motion on the shortest ones. It therefore enables probing the evolution of the Ornstein-Uhlenbeck process toward the Wiener-like process limit at short times [17-19]. In this paper, we analytically derive our two estimators in such a way that they can comprehensively describe the two regimes. In excellent agreement with analytical results and simulated data, our experiments are able to identify over six decades of time the signatures of the noise and ergodic estimators associated with the distinctive features of both diffusion regimes. This description includes the crossover which is classically seen on motional power spectral densities in optical traps [20] but which demands special care when it comes to assessing noise features and ergodic properties.

Our methodology is general and the estimators can be implemented on optical traps that operate in the underdamped 


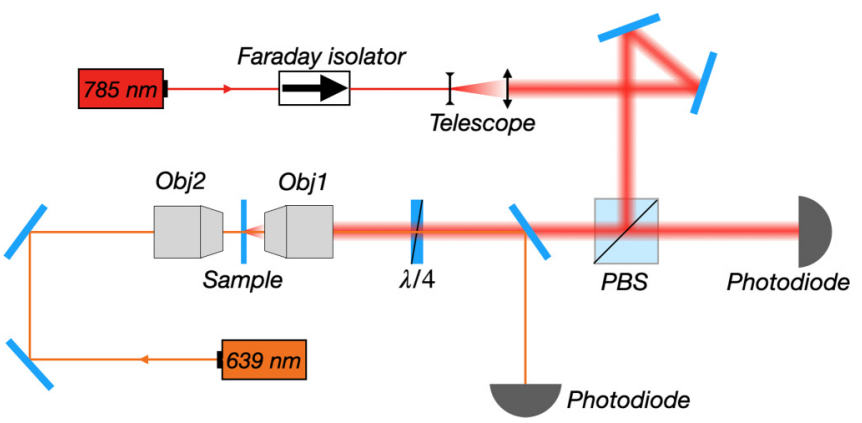

FIG. 1. Schematic view of the experimental setup, displaying the trapping laser (785-nm semiconductor laser, optically isolated and expanded) and the probe laser $(639 \mathrm{~nm})$ used for recording the instantaneous displacement of a $1-\mu \mathrm{m}$ polystyrene bead. The trapping laser is focused inside a fluidic cell in which the bead is immersed using a polarizing beam splitter (PBS) and a water immersion objective $(\mathrm{Obj} 1,60 \times, \mathrm{NA}=1.2)$. The quarter wave-plate $(\lambda / 4)$ ensures that the light scattered by the trapped bead and collected by Obj1 is directed toward the on-axis photodiode. The probe laser illuminates the bead from the backside using a second objective (Obj2, 60×, $\mathrm{NA}=0.7$ ) and is collected by Obj1. A dichroic beamsplitter sends the probe light to the second photodiode that gives the signal used for our analyses.

(inertial) regime. Moreover, these estimators together will turn very useful for characterizing colored noise and nonergodic effects that enrich the physics of more complex Brownian systems.

\section{WIENER VERSUS ORNSTEIN-UHLENBECK CROSSOVER IN AN OPTICAL TRAP}

Free Brownian motion driven only by the Gaussian white noise of thermal fluctuations is described by the Wiener process $W_{t}$. The displacement of the overdamped free Brownian object reads

$$
d x_{t}=\sqrt{2 D} d W_{t},
$$

working directly with the differential $d W_{t}$ with the following properties: $\left\langle d W_{t}\right\rangle=0,\left\langle d W_{t} d W_{t^{\prime}}\right\rangle=\delta\left(t, t^{\prime}\right) d t$. The diffusion coefficient $D=k_{B} T / \gamma$ involves the Boltzmann constant $k_{B}$, the temperature of the surrounding fluid $T$, and the Stokes drag coefficient $\gamma$.

Inside the trap, the harmonic optical potential modifies the stochastic process by exerting on the object a restoring force characterized by a constant stiffness $\kappa$. The same displacement now follows the Ornstein-Uhlenbeck process:

$$
d x_{t}=-\frac{\kappa}{\gamma} x_{t} d t+\sqrt{2 D} d W_{t} .
$$

Our experiment consists of trapping in ultrapure water a single Brownian micron-sized spherical bead in the harmonic potential created at the waist of a focused laser beam and recording in real time the overdamped position of the trapped bead. The experimental setup is schematized on Fig. 1 and detailed in Appendix A. The trapping potential is formed by focusing a 785-nm laser beam and the instantaneous position $x(t)$ is recorded using a second 639-nm low-power laser acting as a probe, as shown in Fig. 1. All the experimental results pre-
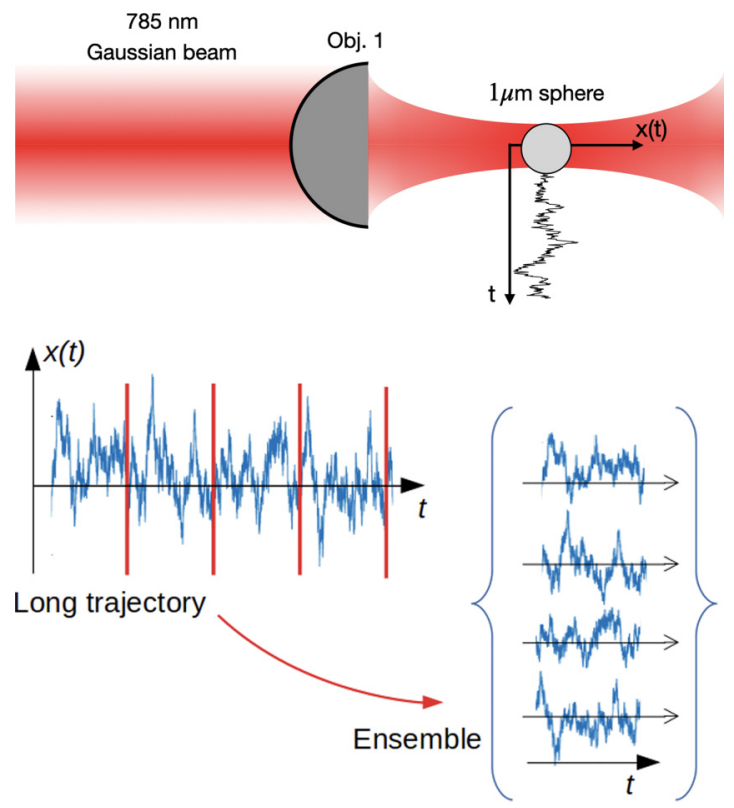

FIG. 2. The instantaneous position of the bead trapped at the laser waist is recorded along the optical axis with an acquisition frequency of $2^{15}=32768 \mathrm{~Hz}$ for $10 \mathrm{~min}$. The result is a very long trajectory that can be used as such. It can also be recombined into an ensemble of shorter trajectories, as schematized. In our experiments, the thermally limited axial displacement $\delta z \sim \sqrt{k_{\mathrm{B}} T / \kappa} \sim 40 \mathrm{~nm}$ of the bead taking the values extracted below in Fig. 3 is fully contained within the Rayleigh range $\sim 0.5 \mu \mathrm{m}$ of the 639 -nm laser, leading to a linear relation between the intensity modulation and the axial displacement. Corresponding calibration procedures are described in Ref. [9].

sented in this paper are obtained from a 10-min-long trajectory (i.e., $1.97 \times 10^{7}$ successive position measurements acquired at an acquisition frequency of $2^{15}=32768 \mathrm{~Hz}$ ). As noted in the Introduction, this long trajectory can be used as a whole, for example, in high-sensitivity measurements or can also be cut and rearranged, for instance in stochastic thermodynamics studies, in an ensemble of subtrajectories if the system is ergodic. The procedure is sketched in Fig. 2 and relies on the fact that the modulation of the $639-\mathrm{nm}$ light is proportional to the axial displacement of the bead inside the trap.

These data are compared, throughout this paper, with numerical simulations obtained from an algorithm for the Wiener process,

$$
x_{t+\Delta t}=x_{t}+\sqrt{2 D \Delta t} \theta_{t},
$$

where $\theta$ is a dimensionless Gaussian white noise with $\left\langle\theta_{t}\right\rangle=$ $0,\left\langle\theta_{t} \theta_{t^{\prime}}\right\rangle=\delta\left(t-t^{\prime}\right)$, according to the methods detailed in Ref. [21]. By the same token, the algorithm for the OrnsteinUhlenbeck process is

$$
x_{t+\Delta t}=x_{t}-\frac{\kappa}{\gamma} x_{t} \Delta t+\sqrt{2 D \Delta t} \theta_{t} .
$$

This discretization method, known as the Euler-Maruyama method, corresponds to an $\mathcal{O}\left(\Delta t^{1 / 2}\right)$ approximation of ItôTaylor expansions [22]. As discussed in detail in Appendix D, higher order terms lead to a more efficient algorithm known as the Mildstein algorithm, which our simulations are based 


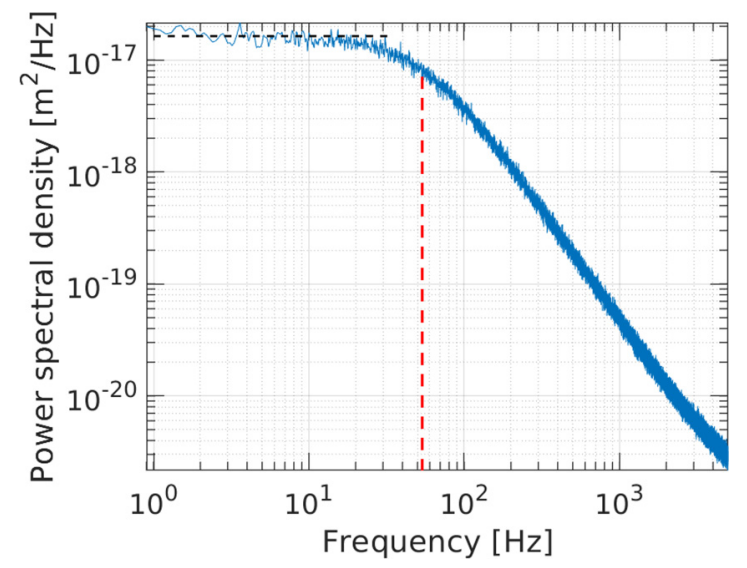

FIG. 3. Experimental power spectrum density (PSD) evaluated for a trajectory $x(t)$ measured from $0.03 \mathrm{~Hz}$ to $100 \mathrm{kHz}$, displaying a large signal-to-noise ratio, spanning over four decades. We also mark with a vertical red dashed line the transition at the roll-off frequency $(53.6511 \mathrm{~Hz})$ between the high-frequency almost-free regime and the low-frequency trapped regime. The thermal noise plateau $4 k_{B} T \gamma / \kappa$ (horizontal black dashed line) agrees well with the low-frequency limit of the PSD, as expected. From the Lorentzian fit performed on the PSD, we can extract the stiffness $\kappa=2.9614 \pm 0.0673 \mathrm{pN} / \mu \mathrm{m}$. The experiments are performed at room temperature, $T \approx 295 \mathrm{~K}$ and the $1-\mu \mathrm{m}$ bead experiences a drag coefficient $\gamma=6 \pi \eta R \mathrm{~kg} / \mathrm{s}$ where $\eta \approx 0.95 \times 10^{-3}$ Pa s, hence $\gamma=8.9837 \times 10^{-9} \mathrm{~kg} / \mathrm{s}$. These parameters, with the stiffness extracted from the Lorentzian fit of the PSD, are used in all numerical and analytical results done throughout the paper.

on and which converges more quickly toward the analytical expression as $\Delta t$ decreases $[23,24]$.

From Eq. (2), the Brownian motion in the trap can be spectrally analyzed with the position's power spectral density (PSD):

$$
S_{x}(f)=\frac{D}{\pi^{2}\left(f_{c}^{2}+f^{2}\right)} .
$$

As clearly seen on the experimental PSD displayed in Fig. 3 over ca. four decades, the roll-off frequency $f_{c}=\kappa /(2 \pi \gamma)$-measured at $\sim 53 \mathrm{~Hz}$-separates the highfrequency regime $S_{x}(f) \sim D /\left(\pi^{2} f^{2}\right)$ of free Brownian motion-see Eq. (1) - from the low-frequency trapping regime $S_{x}(f) \sim D /\left(\pi^{2} f_{c}^{2}\right)=4 k_{B} T \gamma /\left(\kappa^{2}\right)$ - see Eq. (2). The PSD thus clearly reveals how a Wiener regime corresponds in the optical trap to the short time $\delta t \ll \gamma / \kappa$ limit of the Ornstein-Uhlenbeck process (in other words, when observed over such a short timescale, the Brownian object moves inside the trap as if it were freely diffusing without confinement).

\section{NOISE STABILITY: ALLAN VARIANCE AND STATISTICAL TESTS}

To characterize the noise at play inside the optical trap, it is central to measure two of its properties: its nature (color, thermal weight, frequency contributions, etc.), and its stability in time. Testing the nature of the noise can be done spectrally with the PSD that yields the different frequency contributions of the noise. Integrated PSD can also reveal the

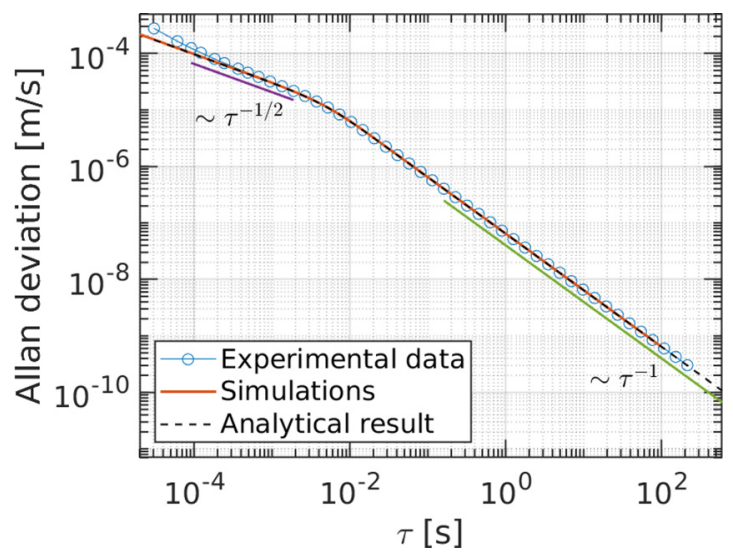

FIG. 4. Allan standard deviation evaluated for the long trajectory experimentally recorded (blue open circles). We plot the simulated Allan standard deviation (orange continuous line) superimposed to the analytical result (black dashed line). We highlight the slopes in both free (purple continuous line) and trapped regimes (green continuous line). We observe that the whole time range experimentally accessible from $\sim 10^{-4} \mathrm{~s}$ up to $\sim 10^{2} \mathrm{~s}$ and that spans more than six decades of time is perfectly captured by the theoretical expression built with experimental parameters $-\gamma, T$, $\kappa$, see Fig. $3-$ with very good agreement. The small departure of the experimental data from the theoretical Allan variance is attributed to tracking errors discussed in Appendix H3.

thermal nature of the noise through the fluctuation-dissipation theorem. However, the spectral approach turns out to be exposed to possible low frequency drifts that can modify noise properties $[10,25,26]$. To avoid this stability issue, we work in the time domain and perform an Allan-variance-based test of the system, capable of revealing low-frequency drifts within a stochastic signal $[27,28]$. This approach leads us to verify unambiguously the stationary and thermally limited properties of the noise at play in an experiment.

The Allan variance $\sigma^{2}(\tau)$ can be connected to the noise $\operatorname{PSD} S(f)$ through the following relation [28]:

$$
\sigma^{2}(\tau)=\frac{4}{\pi \tau^{2}} \int_{-\infty}^{+\infty} S(f) \sin ^{4}(\pi f \tau) d f .
$$

It can therefore be explicitly evaluated analytically for the Ornstein-Uhlenbeck PSD $S_{x}(f)$ of Eq. (5),

$$
\sigma^{2}(\tau)=\frac{k_{B} T}{\kappa \tau^{2}}\left(4\left[1-e^{-\kappa \tau / \gamma}\right]-\left[1-e^{-2 \kappa \tau / \gamma}\right]\right),
$$

as detailed in Appendix E.

The experimental Allan variance is shown in Fig. 4 following the same methodology presented in our earlier work [10]. This experimental Allan variance is compared with numerical simulations and with the analytical result of Eq. (7). We note a remarkable experiment-theory agreement over more than six decades in time. These results show the very high level of noise stability up to $>250 \mathrm{~s}$ that one can reach on a simple optical trap setup such as ours.

However, they also reveal how the Ornstein-Uhlenbeck and the Wiener processes are characterized by different Allan variance signatures. Indeed, we identify here two clear asymptotic regimes. The short-time regime $(\tau \ll \gamma / \kappa)$ falls 


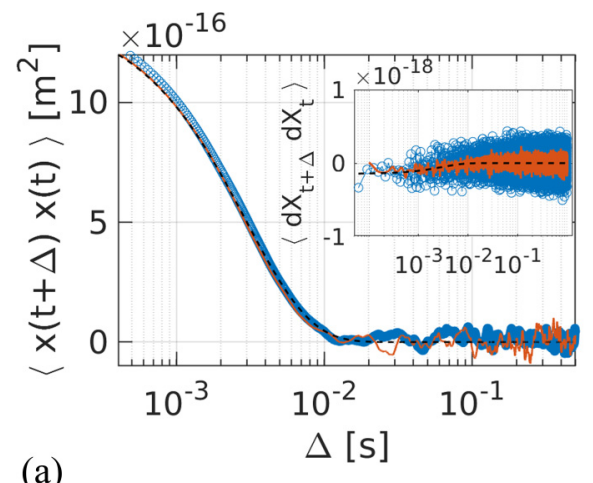

(a)

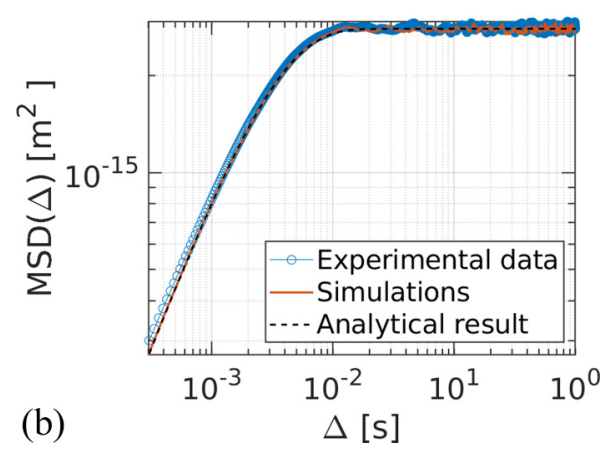

FIG. 5. (a) Time-averaged covariances of positions and displacements (inset). Experimental data are plotted (blue open circles) together with the simulation results (orange continuous line) and the analytical prediction (black dashed line). (b) Comparison between the measured mean square displacements (MSDs) (blue open circles) and the analytical expression given in Eq. (9) obtained in the stationary regime (black dashed line). The comparison with simulation results is also displayed (orange continuous line). The very good agreement with both theory and simulations shows that the measured process can be considered as stationary. We note the same relaxation time of $3 \times 10^{-3} \mathrm{~s}$ for all data, revealing the crossover between the free (Wiener) and trapped (Ornstein-Uhlenbeck) diffusion regimes. Again, the small departure of the experimental data with respect to the theoretical MSD is attributed to tracking errors discussed in Appendix $\mathrm{H} 2$.

on the $\sigma_{\text {free }} \sim t^{-1 / 2}$ slope, which is known to correspond to the thermal white noise limit of free Brownian motion $[10,25]$. Interestingly, in the long-time limit $(\tau \gg \gamma / \kappa)$ of the Ornstein-Uhlenbeck process where the trapping action dominates the motional dynamics, the Allan variance shows a different slope with $\sigma_{\text {trap }} \sim t^{-1}$. This change of signatures between the two regimes, accounting for the presence of the harmonic force field in the long-time limit, is continuous. We observe a very good match between the experiments and theory in the transition between asymptotic regimes.

The slight differences at short time-lags between the theory and the experimental data will also be observed at the level of the mean squared displacement (MSD) Fig. 5(a) and the ergodic estimator Fig. 6. As discussed in detail in Appendix $\mathrm{H}$, these deviations are due to tracking errors unavoidably induced experimentally by the photodiode and electronic system used for recording our Brownian trajectories.

We will now use an alternative method based on the autocorrelation and the MSD for identifying either a Wiener

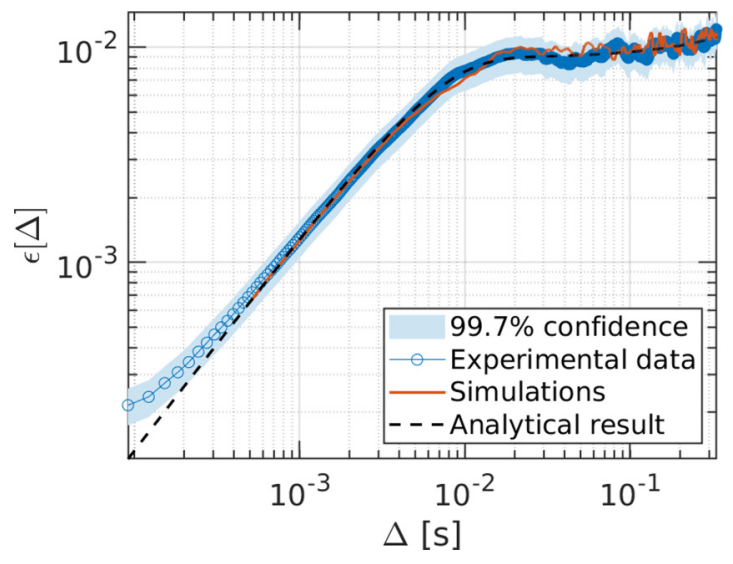

FIG. 6. The normalized variance $\epsilon(\Delta)$ playing the role of an ergodic estimator is displayed (black dashed line) when calculated for the Ornstein-Uhlenbeck process at play in our optical trap. Experimental results (blue open circles) for $\epsilon(\Delta)$ are compared to the theory within a $99.7 \%$ confidence interval. We also show the results of a numerical simulation using $\mathcal{O}(3 / 2)$ algorithm (orange continuous line). The slight deviation at short times between the experiment and the theory comes again mainly from the position tracking errors whose impact on the ergodic estimator is discussed in Appendix H4.

or an Ornstein-Uhlenbeck process. We, however, note here that at thermal equilibrium, Wiener and Ornstein-Uhlenbeck processes generate trajectories $x(t)$ with different statistical properties. Indeed, the Ornstein-Uhlenbeck process of the trapped Brownian motion has a variance constant in time with the equipartition condition $\left\langle x_{t}^{2}\right\rangle=k_{B} T / \kappa$. In contrast, the Wiener process of free Brownian motion is nonstationary with a motional variance that grows linearly in time, but looking at the statistical properties of successive displacements $d x_{t}$ whose dynamics is governed by Eqs. (1) and (2), it becomes possible to perform the same stationarity test for both processes. To do that, we will use the autocorrelation of displacements and the MSD, extracted from long trajectories. We will verify stationarity-in the strong sense since the noise is Gaussian-with i a fixed mean (that can be removed without any loss of generality), (ii) a finite variance $\overline{d x_{t}^{2}}$, and (iii) a displacement covariance (autocorrelation) $\overline{d x_{t} d x_{s}}$ that depends only on the absolute time difference $\Delta=|t-s|$.

The covariance of displacements can be computed using Eq. (2) [details are given in Appendix B, see Eq. (B9)] and yields

$$
\overline{d x_{t} d x_{s}}=-\frac{2 \kappa k_{B} T}{\gamma^{2}} e^{-\kappa|t-s| / \gamma} d t^{2}+2 D \delta(t-s) d t .
$$

This theoretical expression is compared to the covariance evaluated experimentally as a time average on successive displacements. The comparison, together with simulations, is shown in Fig. 5(a). The convergence of the time-averaging process for the covariance toward the theoretical expression, only function of $\Delta$, shows the absence of dependence on the absolute time $t$.

We can also evaluate the MSD directly from the measurement of successive positions separated by a given time lag $\Delta$ 
[details are given in Appendix C, see Eq. (C4)] as

$$
\overline{\delta x^{2}(\Delta)}=2 \frac{k_{B} T}{\kappa}\left(1-e^{-\kappa \Delta / \gamma}\right)
$$

Again, this theoretical result is compared to the experimental MSD which is given by evaluating the time-averaged MSD of the entire trajectory. The comparison, also including simulations, shows very good agreement displayed in Fig. 5(b).

This agreement, together with the covariance, depending only on time difference, confirms that our Brownian trap implements a strong stationary Ornstein-Uhlenbeck process. Clearly, our data demonstrate a smooth crossover between the linear MSD at short time lags associated with a Wiener regime and the constant MSD at longer time lags that reflects the confined nature of the diffusion for the Ornstein-Uhlenbeck process.

\section{TEST OF ERGODICITY}

We already stressed in the Introduction the crucial importance of verifying the ergodic hypothesis when the long trajectory is analyzed through an ensemble of its parts (subtrajectories). Ergodicity per se corresponds to the equality taken in the infinite time limit $\mathcal{T} \rightarrow \infty$, between the time average and the ensemble average for a given stochastic process. Since the ensemble of subtrajectories $\{j\}$ is built from parts taken at different times of the initial long trajectory as shown in Fig. 2, any statistical analysis performed on this ensemble is based on the ergodic hypothesis. When one is using a specific rearrangement for a given experiment, ergodicity must be verified on this ensemble. To illustrate this, we reshape our long trajectory into an ensemble of 600 subtrajectories $x_{i}(t)$ of 1 -s duration each. Note that we carefully show in Appendix F that the choice of the length of the subtrajectories (and hence the size of the ensemble) does not change the validity of the test.

For a trajectory $x_{i}(t)$ drawn from the ensemble, ergodicity is defined as

$$
\lim _{\mathcal{T} \rightarrow \infty} \frac{1}{\mathcal{T}} \int_{0}^{\mathcal{T}} x_{i}(t) d t=\left\langle x_{j}(t)\right\rangle_{\{j\}}
$$

Although simple, this definition is, however, hardly operative in experiments that only yield ensembles of finite-time trajectories. Following the approach proposed in Refs. $[15,16]$, we prefer resorting to an estimator that can characterize the ergodic nature of an experiment performed over a finite integration time. This estimator is grounded on the stationary nature of the MSD which is, as shown above, independent of the choice from the initial time and only depends on the time lag $\Delta$. In such conditions, ergodicity simply demands the time-averaged MSD of any ith-trajectory, as defined above, to be equal, in the long $\mathcal{T} / \Delta$ limit, to the ensemble mean of individual time average taken over the ensemble $\{j\}$ of available trajectories:

$$
\lim _{\mathcal{T} / \Delta \rightarrow \infty} \overline{\delta x_{i}^{2}(\Delta)}=\overline{\left\langle x_{j}^{2}(\Delta)\right.} \mid
$$

Formally, ergodicity demands that the $\overline{\delta x_{i}^{2}(\Delta)} /\left\langle\overline{\delta x_{i}^{2}(\Delta)}\right\rangle$ ratio tends to a Dirac distribution as $\mathcal{T} / \Delta \rightarrow \infty$. A sufficient condition for ergodicity is therefore that the normalized variance of this ratio goes to zero in the limit $\mathcal{T} / \Delta \rightarrow \infty$ :

$$
\left.\epsilon(\Delta)=\frac{\left\langle{\overline{\delta x_{i}^{2}(\Delta)}}^{2}\right\rangle-\left\langle\left.\overline{\delta x_{i}^{2}(\Delta)}\right|^{2}\right.}{\left\langle\delta x_{i}^{2}(\Delta)\right.}\right\rangle^{2} .
$$

Therefore, handling finite integration times, this normalized variance $\epsilon(\Delta)$ is a valid estimator to prove the ergodic nature of a stochastic process experimentally implemented. One can choose to study $\epsilon$ as a function of the total integrated time $\mathcal{T}$, with fixed $\Delta$. We do this in Appendix F. Here, rather, we show in Fig. 6 the evolution of $\epsilon$ as a function of the time lag $\Delta$ because this choice reveals different signatures for each of the two regimes of free and trapped diffusion inside the optical trap.

One very appealing aspect of $\epsilon(\Delta)$ is that it can be theoretically calculated for an Ornstein-Uhlenbeck process, as we do in Appendix G. This gives the capacity to characterize the ergodicity throughout the spectral range of the optical trap, therefore both in the long-time trapped and the short-time free diffusion regimes. These two regimes correspond to different time-lag evolutions of $\epsilon(\Delta)$, as clearly seen in Fig. 6. Here too, a smooth crossover between the long time-lag trapped (Ornstein-Uhlenbeck) regime and the short time-lag free (Wiener limit) regimes is revealed and measured, with the transition time-lag determined from the trap stiffness, as discussed in more detail in Appendix G. The experimental evolution of $\epsilon(\Delta)$ corresponding to the recorded finite-time trajectories obtained for our trapping experiment is also shown. The excellent agreement with the theoretical $\epsilon(\Delta)$ in both the freely diffusing and in the trapped regimes confirms that our optical trapping process can be considered ergodic with a high level of confidence. Because $\epsilon(\Delta)$ is formally a variance, the quality of its estimator on a finite-size ensemble can be quantified using a $\chi^{2}$ test. We perform this test in Fig. 6 up to a $3 \sigma$ level of confidence.

\section{CONCLUSION}

By implementing Allan variance-based, stationarity, and ergodic tests together using appropriate estimators, we have been able to fully characterize, through wide spectral ranges, the nature of the noise and the ergodicity of the stochastic regimes at play in an overdamped optical trap. In particular, our estimators have distinctive features between the highand low-frequency range of the trap that we have described analytically, including the crossover between the two freeand trapped-diffusion regimes. This led us to discuss clear differences from the viewpoint of noise and ergodicity assessments between the trapped regime of the Ornstein-Uhlenbeck process and its Wiener-like limit, notwithstanding that they are driven by the same Gaussian white thermal noise. In stochastic thermodynamics, ergodic processes are a very important subclass of stationary processes. When aiming to exploit Brownian systems, it is therefore very important to be able to identify stationarity signatures. The simple and straightforward methodology proposed in our paper is also relevant to many recent experiments involving Brownian 
systems coupled to nonthermal, colored, and more complex noise environments [29,30].

\section{ACKNOWLEDGMENTS}

Thanks are due to A. Canaguier-Durand and G. Schnoering for stimulating discussions. This work of the Interdisciplinary Thematic Institute QMat, as part of the ITI 2021 2028 program of the University of Strasbourg, CNRS and Inserm, was supported by IdEx Unistra (ANR-10-IDEX0002) and by SFRI STRATUS project (ANR-20-SFRI-0012), and by ANR Equipex Union (ANR-10-EQPX-52-01), the Labex NIE projects ANR-11-LABX-0058_NIE, and USIAS (ANR-10-IDEX-0002-02) under the framework of the French Investments for the Future Program.

\section{APPENDIX A: EXPERIMENTAL SETUP}

Our experiment consists of trapping in ultrapure water a single Brownian object in the harmonic potential created at the waist of a focused laser beam. A schematic view of the setup is given in Fig. 1. A linearly polarized Gaussian beam (OBIS Coherent, CW $785 \mathrm{~nm}, 110 \mathrm{~mW}$ ) is focused by a water immersion objective (Nikon Plan Apochromat $60 \times$, Numerical Aperture 1.20) into the sample that consists of a cell made of a glass slide and a coverslip, separated by a $120-\mu \mathrm{m}$-thick and 1-cm-wide spacer. The cell is filled with a colloidal dispersion of polystyrene microspheres (ThermoFisher FluoSpheres polystyrene microspheres, $1-\mu \mathrm{m}$ diameter $\pm 2 \%$ ) diluted in deionized water. We start with a solution of concentration of $10^{10}$ beads $/ \mathrm{mL}$ that we dilute $\sim 10^{5} \times$. The cell is then taped to a metallic holder mounted in our optical setup.

The instantaneous position of the trapped single bead is recorded using an additional low-intensity counterpropagating laser beam (Thorlabs HL6323MG CW 639 nm, 30 mW, but here used at low power), focused on the bead using a second objective (Nikon Plan-fluo Extra Large Working Distance $60 \times$, Numerical Aperture 0.7). Within the small trapping volume defined by our setup, the intensity of the light scattered by the microsphere scales linearly with its displacement $x(t)$ along the optical axis. This scattered intensity signal is collected through the trapping objective and sent to a P.I.N. photodiode (Thorlabs, model Det10A2). The output signal recorded in $\mathrm{V}$ is sent to a low noise amplifier (Stanford Research, SR560) and then acquired by an analog-to-digital card (National Instrument, PCI-6251). The signal is filtered through a $0.3-\mathrm{Hz}$ high-pass filter at $6 \mathrm{~dB} /$ oct to remove the DC component of the output signal and through a $100-\mathrm{kHz}$ low-pas filter at $6 \mathrm{~dB} /$ oct to prevent aliasing. Finally, we convert the voltage signal into a position in meter following the approach we presented in Ref. [9].

In our experiment, it is crucial to trap only one bead at a time. To achieve this, we rely on (i) a low concentration of beads in the solution and (ii) a direct imaging of the vicinity of the trap with an interferometric scattering microscopy technique (not shown in the figure but presented in detail in our previous work [31]). A second important point is ensured by the thickness $(120 \mu \mathrm{m})$ of the cell: The trapping region must be localized far enough from the walls as to keep fluid parameters constant. The choice of the trapping wavelength $(785 \mathrm{~nm})$ also avoids locally heating the fluid. The data presented in the paper are taken from ten consecutive measurements of $60 \mathrm{~s}$ each, with an acquisition frequency of $32768 \mathrm{~Hz}$. The whole experiment is done in constant conditions, with the same bead and only a few seconds between each measurement. It is thermalized with a precision $\pm 1 \mathrm{~K}$ at room temperature and well isolated from spurious noise sources, leading to measure stable signals over six decades of times with long time-series of 19660800 positions, spanning over $10 \mathrm{~min}$. The concatenation of ten measurements leads to ten discontinuities among the 19660800 points. However, the motion being confined, these discontinuities are of the same order of magnitude than a regular increment. This together with the small number of such cuts among a large statistics prevent any statistical contribution that would modify the results.

\section{APPENDIX B: AUTOCORRELATION OF DISPLACEMENT}

We will compute the autocorrelation function (or covariance, since the process has zero mean) of displacements $d X_{t}$ defined by the Ornstein-Uhlenbeck process $d X_{t}=-a X_{t} d t+$ $b d W_{t}$ (adopting simple notations $\kappa / \gamma \equiv a$ and $\sqrt{2 k_{B} T / \gamma} \equiv$ b) as

$$
\begin{aligned}
\left\langle d X_{t} d X_{s}\right\rangle= & \left\langle\left(-a X_{t} d t+b d W_{t}\right)\left(-a X_{s} d s+b d W_{s}\right)\right\rangle \\
= & \underbrace{a^{2}\left\langle X_{t} X_{s}\right\rangle d t^{2}}_{(1)}-\underbrace{a b\left\langle X_{t} d t d W_{s}\right\rangle}_{(2)} \\
& -\underbrace{a b\left\langle X_{s} d s d W_{t}\right\rangle}_{(3)}+\underbrace{b^{2}\left\langle d W_{t} d W_{s}\right\rangle}_{(4)} .
\end{aligned}
$$

Using the solution of the Ornstein-Uhlenbeck process,

$$
X_{t}=X_{0} e^{-a t}+b e^{-a t} \int_{0}^{t} e^{a t^{\prime}} d W_{t^{\prime}},
$$

and assuming that all time increments are equal $(\forall t, s: d t=$ $d s$ ), we can compute the different terms in (B1) one by one,

$$
\text { (1) }=\frac{a b^{2}}{2} e^{-a|t-s|} d t^{2}
$$

since at equilibrium $\left\langle X_{0}^{2}\right\rangle=k_{B} T / \kappa=b^{2} / 2 a$ [see below Eq. (C3)],

$$
\begin{aligned}
(2) & =-a b\left\langle X_{t} d t d W_{s}\right\rangle \\
& =-a b\left\langle X_{0} d W_{s}\right\rangle e^{-a t} d t-a b^{2} d t \int_{0}^{t} e^{a\left(t_{1}-s\right)}\left\langle d W_{t_{1}} d W_{s}\right\rangle \\
& =-a b \delta(s-0) d t^{2} e^{-a t}-a b^{2} d t \int_{0}^{t} e^{a\left(t_{1}-s\right)} \delta\left(t_{1}-s\right) d s .
\end{aligned}
$$

If we consider nonzero times, we can ignore the first term. For the second, we have two cases:

$$
(2)= \begin{cases}-a b^{2} d t^{2} e^{-a(t-s)} & \text { if } t \geqslant s \\ 0 & \text { if } t<s\end{cases}
$$

similarly,

$$
\text { (3) }= \begin{cases}0 & \text { if } t>s \\ -a b^{2} d t^{2} e^{-a(s-t)} & \text { if } t \leqslant s\end{cases}
$$




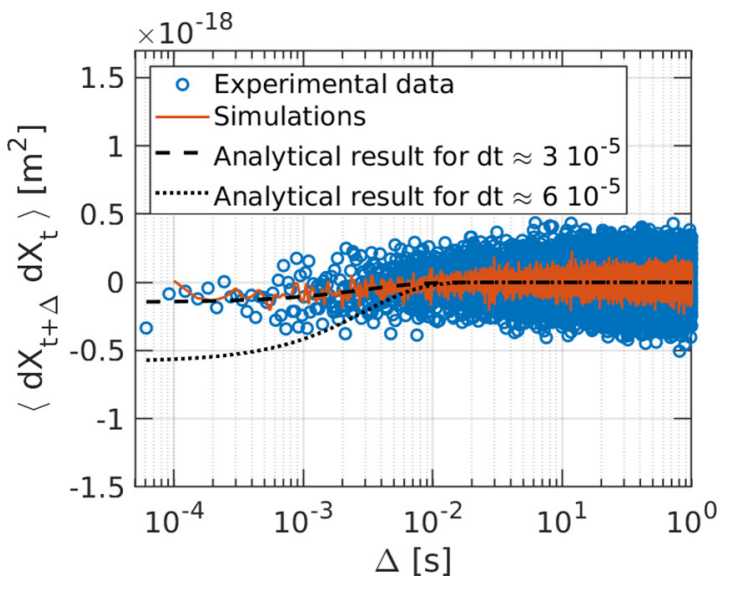

FIG. 7. Covariance of displacements for the Ornstein-Uhlenbeck process. We plot the experimental result (blue open circles), calculated with $d t=3.0518 \times 10^{-5} \mathrm{~s}$ along with simulation result (orange continuous line) and analytical solution (B9) (black continuous line). We plot (black dashed and continuous lines) the analytical result for two different values of the time step $d t$ to highlight the fact that the deviation from zero of the Ornstein-Uhlenbeck displacements is strongly dependent on the value of $d t$, converging rapidly to zero with increasing acquisition frequency.

We can therefore combine them into $(2)+(3)=$ $-a b^{2} d t^{2} e^{-a(\max (t, s)-\min (t, s))}$, giving

$$
(2)+(3)=-a b^{2} e^{-a|t-s|} d t^{2} \text {. }
$$

For the fourth term, we have simply

$$
\text { (4) }=b^{2} \delta(t-s) d t \text {, }
$$

that vanishes if $t \neq s$. These four terms added together lead to the simple expression of the autocorrelation of displacements:

$$
\left\langle d X_{t} d X_{s}\right\rangle=-\frac{a b^{2}}{2} e^{-a|t-s|} d t^{2}+b^{2} \delta(t-s) d t .
$$

Putting back physical dimensions with $a b^{2} d t^{2}=\frac{2 \kappa k_{B} T}{\gamma^{2}} d t^{2}$ and $\beta^{2} d t=\frac{2 k_{B} T}{\gamma} d t$ (both in $\left[\mathrm{m}^{2}\right]$ ), we get

$$
\left\langle d X_{t} d X_{s}\right\rangle=-\frac{2 \kappa k_{B} T}{\gamma^{2}} e^{-\kappa|t-s| / \gamma} d t^{2}+2 D \delta(t-s) d t .
$$

Since $\left\langle d W_{t} d W_{s}\right\rangle=\overline{d W_{t} d W_{s}}$ for a Wiener process [19], we can identify the ensemble average $\left\langle d X_{t} d X_{s}\right\rangle$ with a time averaged covariance $\overline{d X_{t} d X_{s}}$ that is experimentally measured-see Eq. (8) in the main text-and displayed in Fig. 5 in the main text and in Fig. 7 here.

Figure 7 reveals good agreement between the experimental results, the simulations, and the theoretical result (B9). The covariance converges toward zero (which is the covariance of the Wiener increment) for decreasing $d t$. However, the nondifferentiability of the stochastic process prevents us from taking the limit of vanishingly small $d t$ and from observing the convergence of the short-time Ornstein-Uhlenbeck process toward a Wiener-like process.

\section{APPENDIX C: DERIVATION OF THE MEAN SQUARE DISPLACEMENT}

Using the general solution of the Ornstein-Uhlenbeck stochastic differential equation,

$$
x_{t}=x_{0} e^{-\kappa t / \gamma}+\sqrt{2 D} e^{-\kappa t / \gamma} \int_{0}^{t} e^{\kappa t^{\prime} \gamma} d W_{t^{\prime}},
$$

we write the expression of the autocorrelation function,

$$
\begin{aligned}
\left\langle x\left(t_{1}\right) x\left(t_{2}\right)\right\rangle= & \left(\left\langle x_{0}^{2}\right\rangle-\frac{k_{B} T}{\kappa}\right) e^{-\kappa\left(t_{1}+t_{2}\right) / \gamma} \\
& +\frac{k_{B} T}{\kappa} e^{-\kappa\left|t_{1}-t_{2}\right| / \gamma}
\end{aligned}
$$

that simplifies into

$$
\left\langle x\left(t_{1}\right) x(t 2)\right\rangle=\frac{k_{B} T}{\kappa} e^{-\kappa\left|t_{1}-t_{2}\right| / \gamma}
$$

if $\left\langle x_{0}^{2}\right\rangle=\frac{k_{B} T}{\kappa}$,i.e., if the initial distribution is at equilibrium. The MSD therefore reads

$$
\begin{aligned}
\left\langle\delta x^{2}(\Delta)\right\rangle & \equiv\left\langle(x(t+\Delta)-x(t))^{2}\right\rangle \\
& =\left\langle x(t+\Delta)^{2}\right\rangle-2\langle x(t+\Delta) x(t)\rangle+\left\langle x(t)^{2}\right\rangle \\
& =\frac{k_{B} T}{\kappa}-2 \frac{k_{B} T}{\kappa} e^{-\kappa \Delta / \gamma}+\frac{k_{B} T}{\kappa},
\end{aligned}
$$

that is,

$$
\left\langle\delta x^{2}(\Delta)\right\rangle=2 \frac{k_{B} T}{\kappa}\left(1-e^{-\kappa \Delta / \gamma}\right) .
$$

Using the same property of the Wiener process used in Appendix B, one has $\left\langle\delta x^{2}(\Delta)\right\rangle=\overline{\delta x^{2}(\Delta)}$, allowing us to compare Eq. (C4) to the experimental result given in Eq. (9) in the main text.

\section{APPENDIX D: BROWNIAN MOTION SIMULATIONS}

This Appendix briefly presents the structure of the stochastic algorithm, as well as the detailed scheme used for the simulations performed in this paper. The general framework is based on an Itô-Taylor expansion, generalizing to stochastic differential equations and standard Taylor expansion procedures [22]. First, for an ordinary differential equation

$$
d X_{t}=a\left[X_{t}\right] d t
$$

and for a function $f\left[X_{t}\right]$, we can use the standard chain rule and write $d f\left[X_{t}\right]=a\left[X_{t}\right] \frac{\partial}{\partial t} f\left[X_{t}\right] d t$. This leads to an integral form

$$
f\left[X_{t}\right]=f\left[X_{0}\right]+\int_{0}^{t} a\left[X_{s}\right] \frac{\partial f\left[X_{s}\right]}{\partial s} d s
$$

that can be truncated at a specified order to approximate the process described. In the case of a stochastic process with the following generic form:

$$
d X_{t}=a\left[X_{t}\right] d t+b\left[X_{t}\right] d W_{t},
$$

where $d W_{t}$ is the stochastic Wiener increment defined by $\left\langle d W_{t}\right\rangle=0,\left\langle d W_{t}^{2}\right\rangle=d t$, we use Itô's lemma instead of the standard chain rule. 
We define $\mathcal{L}^{0}=a_{t} \frac{\partial}{\partial X}+\frac{1}{2} b_{t}^{2} \frac{\partial^{2}}{\partial X^{2}}$ and $\mathcal{L}^{1}=b_{t} \frac{\partial}{\partial X}$ and use the following notations: $a_{t} \equiv a\left[X_{t}\right]\left(\right.$ simil. $\left.b_{t}\right)$ to keep the notation light. Then, similarly to Eq. (D2), we obtain

$$
f\left[X_{t}\right]=f\left[X_{0}\right]+\int_{0}^{t} \mathcal{L}^{0} f\left[X_{s}\right] d s+\int_{0}^{t} \mathcal{L}^{1} f\left[X_{s}\right] d W_{s} .
$$

We can apply this to $X_{t}$ itself before iteratively applying it to the quantities $a_{t}$ and $b_{t}$ inside the integrals. Doing so, we obtain successive approximations of the process $f\left[X_{t}\right]$ up to a specified order. Hence for the process $X_{t}$ on a time interval $\Delta t$, an approximation can be given by

$$
X_{t+\Delta t}=X_{t}+a_{t} \int_{t}^{t+\Delta t} d s+b_{t} \int_{t}^{t+\Delta t} d W_{s}+\mathcal{O}\left(\Delta t^{1}\right)
$$

By iterating the same procedure up to higher orders, we obtain algorithms with better precision for a given time increment $\Delta t$. The different terms can be written concisely with the following integrals:

$$
\begin{aligned}
& \Delta t=\int_{t}^{t+\Delta t} d s, \\
& \Delta W=\int_{t}^{t+\Delta t} d W, \\
& \Delta Z=\int_{t}^{t+\Delta t}\left[\int_{t}^{S} d W\right] d S .
\end{aligned}
$$

With these these definitions, the first-order truncation (D5) gives rise to the Euler-Maruyama scheme for $Y_{i}$ taken as the numerical approximation of $X_{t}$,

$$
Y_{i+1}=Y_{i}+a_{i} \Delta t+b_{i} \Delta W_{i}
$$

and where the Wiener increment can be simulated by $\Delta W=$ $\eta \sqrt{\Delta t} \equiv \mathcal{N}(0,1) \sqrt{\Delta t}$. Here, the normally distributed random number can be produced by various means, often using built-in functions for random number generation. In our case, the function used is based on the Box-Muller algorithm.

To evaluate the quality of this algorithm, we rely on the criterion of weak convergence [23], i.e., convergence of the means. We say that an algorithm has a weak order of convergence $n$ is there exists a constant $C$ such that for all function $f\left(X_{t}\right)$ :

$$
\left|\mathbb{E} f\left(X_{t}\right)-\mathbb{E} f\left(Y_{i}\right)\right| \leqslant C \Delta t^{n} .
$$

In our case, we will use $f\left(X_{t}\right)=X_{t}^{2}$ and compare the resulting sample variance to its theoretical value. The Euler-Maruyama algorithm is known to converge with weak order $n=1$. We show in Fig. 8 the results of the weak convergence test, giving an exponent $n_{\text {meas }}=1.1748$.

By the same token, a second-order algorithm can be built by keeping the following terms. This gives the following scheme (derived in Ref. [22]):

$$
\begin{aligned}
Y_{i+1}= & Y_{i}+a_{i} \Delta t+b_{i} \Delta W_{i}+\frac{1}{2} b_{i} b_{i}^{\prime}\left(\Delta W_{i}^{2}-\Delta t\right) \\
& \times a_{i}^{\prime} b_{i} \Delta Z+\frac{1}{2}\left(a_{i} a_{i}^{\prime}+\frac{1}{2} b_{i}^{2} a_{i}^{(2)}\right) \Delta t^{2} \\
& +\left(a_{i} b_{i}^{\prime}+\frac{1}{2} b_{i}^{2} b_{i}^{(2)}\right)(\Delta W \Delta t-\Delta Z) \\
& +\frac{1}{2} b_{i}\left(b_{i} b_{i}^{(2)}+\left(b_{i}^{\prime}\right)^{2}\right)\left(\frac{1}{3} \Delta W^{2}-\Delta t\right) .
\end{aligned}
$$

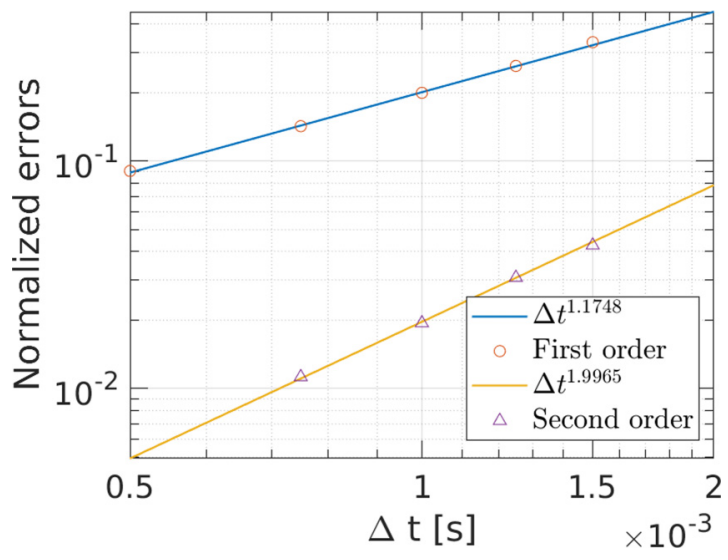

FIG. 8. Weak convergence test of both Euler-Maruyama and second-order algorithms. We plot the errors evaluated as the normalized difference between the measured variance and the theoretical result derived from equipartition $k_{B} T / \kappa$. Namely, $e_{\text {weak }}=\mid 1-$ $\mathbb{E}\left(Y_{i}^{2}\right) /\left(k_{B} T / \kappa\right) \mid$ for different values of the time increment $\Delta t$. We observe that the slopes of $\Delta t^{1.1748}$ and $\Delta t^{1.9965}$ are close to the expected ones of $\Delta t^{1}$ and $\Delta t^{2}$, respectively.

We can now use the fact that the process we are interested in is defined by $a_{t}=\kappa X_{t} / \gamma$ and $b_{t}=\sqrt{2 D}$, which brings all first derivatives of $b_{t}$ and second derivatives of $a_{t}$ to zero. With this simplification, we obtain

$$
Y_{i+1}=Y_{i}+a_{i} \Delta t+b_{i} \Delta W_{i}+b_{i} a_{i}^{\prime} \Delta Z_{i}+a_{i} a_{i}^{\prime} \Delta t^{2} .
$$

As $\Delta W_{i}$ is simulated with a random number $\eta$, it is shown in Ref. [22] that $\Delta Z$ can be simulated using two independent random numbers $\eta$ and $\theta$ and, accordingly,

$$
\begin{aligned}
Y_{i+1}= & Y_{i}+a_{i} \Delta t+b_{i} \sqrt{\Delta t} \eta \\
& +b_{i} a_{i}^{\prime} \frac{1}{2}\left(\eta+\frac{1}{\sqrt{3}} \theta\right) \Delta t^{3 / 2}+a_{i} a_{i}^{\prime} \Delta t^{2} .
\end{aligned}
$$

This is the weak- $\mathcal{O}(2)$ scheme that we have implemented in a PYTHON code to simulate in the main text the ensembles of Brownian trajectories that are compared to experimental data and to the analytical results. This efficient algorithm reduces numerical errors while keeping a reasonable computing cost.

\section{APPENDIX E: ANALYTICAL EXPRESSION OF THE ALLAN VARIANCE FOR THE ORNSTEIN-UHLENBECK PROCESS (HARMONIC POTENTIAL)}

For the Ornstein-Uhlenbeck process given by Eq. (2), we have the following (two-sided) PSD, with $\omega=2 \pi f$ :

$$
S(\omega)=\frac{2 D}{\omega^{2}+\omega_{0}^{2}}
$$

where $D=k_{B} T / \gamma$ is the diffusion coefficient and $\omega_{0}=\kappa / \gamma$ corresponds to the trap roll-off frequency. The Allan variance $\sigma^{2}(\tau)$ is linked to the PSD through a $\sin ^{4}$ transformation, as 


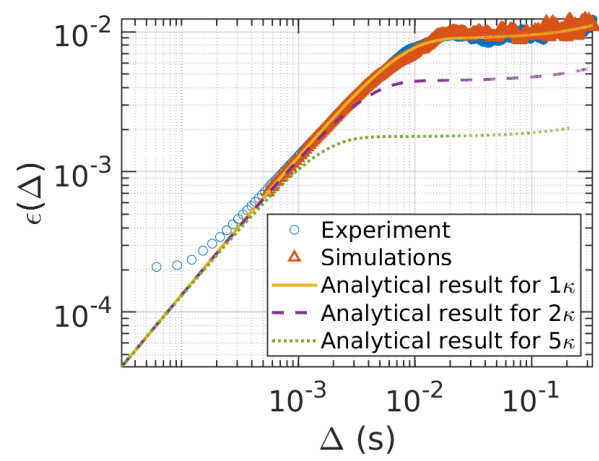

FIG. 9. Ergodic estimator $\epsilon(\Delta)$ analytically calculated-according to Eq. (G16) - for three different stiffnesses (thin lines). The shift of the plateau and the crossover (roll-off) time clearly appears as $\kappa$ increases. The good agreement between theory and experiment is shown for a stiffness of $1 \times \kappa=2.9614 \pm 0.0673 \mathrm{pN} / \mu \mathrm{m}$.

we discussed previously in Ref. [10]:

$$
\sigma^{2}(\tau)=\frac{4}{\pi \tau^{2}} \int_{-\infty}^{+\infty} S(\omega) \sin ^{4}\left(\frac{\omega \tau}{2}\right) d \omega .
$$

With $\sin ^{4}(x)=\left(e^{4 i x}-4 e^{2 i x}+6-4 e^{-2 i x}+e^{4 i x}\right) / 16$ and $\int_{-\infty}^{+\infty}\left(e^{i x}+e^{-i x}\right) d x=2 \int_{-\infty}^{+\infty} e^{i x} d x$ by parity, we write

$$
\sigma^{2}(\tau)=\frac{4}{\pi \tau^{2}} \int_{-\infty}^{+\infty} \frac{2 D}{\omega^{2}+\omega_{0}^{2}} \frac{1}{16}\left(2 e^{2 i \omega \tau}-8 e^{i \omega \tau}+6\right) d \omega,
$$

giving three complex integrals to compute with a simple pole in $\omega= \pm i \omega_{0}$ :

$$
\begin{aligned}
& \int_{-\infty}^{+\infty} \frac{2 e^{2 i \omega \tau}}{\omega^{2}+\omega_{0}^{2}} d \omega=2 i \pi \operatorname{Res}\left(\frac{2 e^{2 i \omega \tau}}{\omega^{2}+\omega_{0}^{2}}, i \omega_{0}\right)=\frac{2 \pi}{\omega_{0}} e^{-2 \omega_{0} \tau}, \\
& \int_{-\infty}^{+\infty} \frac{8 e^{i \omega \tau}}{\omega^{2}+\omega_{0}^{2}} d \omega=2 i \pi \operatorname{Res}\left(\frac{8 e^{i \omega \tau}}{\omega^{2}+\omega_{0}^{2}}, i \omega_{0}\right)=\frac{8 \pi}{\omega_{0}} e^{-\omega_{0} \tau}, \\
& \int_{-\infty}^{+\infty} \frac{6}{\omega^{2}+\omega_{0}^{2}} d \omega=2 i \pi \operatorname{Res}\left(\frac{6}{\omega^{2}+\omega_{0}^{2}}, i \omega_{0}\right)=\frac{6 \pi}{\omega_{0}} .
\end{aligned}
$$

This done, we obtain

$$
\begin{aligned}
\sigma^{2}(\tau) & =\frac{8 D}{\pi \tau^{2}} \frac{1}{16}\left(\frac{2 \pi}{\omega_{0}} e^{-2 \omega_{0} \tau}-\frac{8 \pi}{\omega_{0}} e^{-\omega_{0} \tau}+\frac{6 \pi}{\omega_{0}}\right) \\
& =\frac{k_{B} T}{\kappa \tau^{2}}\left(4\left[1-e^{-\kappa \tau / \gamma}\right]-\left[1-e^{-2 \kappa \tau / \gamma}\right]\right),
\end{aligned}
$$

which corresponds to Eq. (7) in the main text. Two limits are important to draw: (i) the short-time limit $\tau \ll \gamma / \kappa$, where we get $\sigma^{2}(\tau) \approx 2 D / \tau$ corresponding to free Brownian motion [10,25], and (ii) the long-time limit $\tau \gg \gamma / \kappa$ where we get a different behavior $\sigma^{2}(\tau) \approx 3 k_{B} T / \kappa \tau^{2}$.

\section{APPENDIX F: STUDY OF THE ERGODIC ESTIMATOR}

We show in Fig. 9 the impact of the trapping stiffness on the ergodic estimator, clearly displaying how $\kappa$ modifies the longtime plateau as well as the crossover (roll-off) time. We also compare the theory for one specific case with experimental results and numerical simulations.

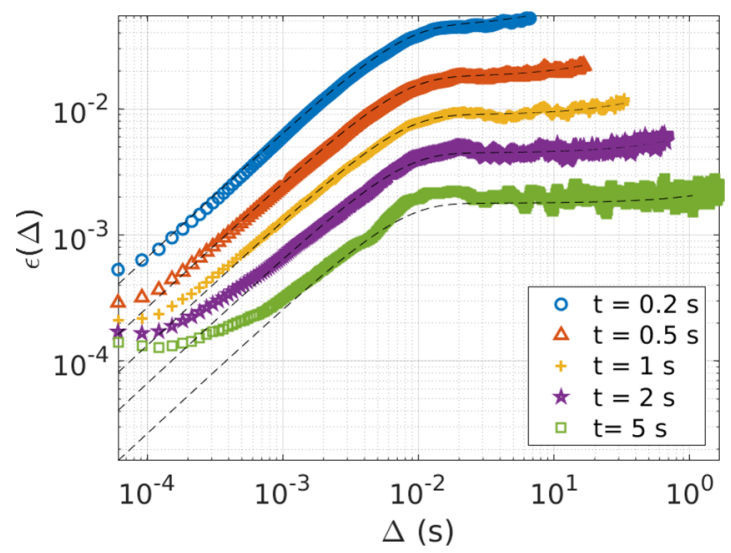

FIG. 10. Experimental ergodic estimator $\epsilon(\Delta)$ for different choices of duration for the subtrajectory arrangements. We plot here the results for 3000 subtrajectories of $0.2 \mathrm{~s}$ (blue), 1200 of $0.5 \mathrm{~s}$ (red), 600 of $1 \mathrm{~s}, 300$ of $2 \mathrm{~s}$, and 120 of $5 \mathrm{~s}$. The short-time deviations from the theoretical curve can be understood as an effect of localization errors discussed in Appendix $\mathrm{H}$.

In the main text, we look at the ergodic estimator for an ensemble of 600 subtrajectories of $t=1 \mathrm{~s}$ duration (length), drawn out of our long trajectory. Other choices of duration are possible and are presented on Fig. 10. We see that the different choices lead to different curves but, within this range, all are in a good agreement with the corresponding analytical result. The only consequence is that for short subtrajectories, the long-time plateau disappears and, for long trajectories, one has less ensemble statistics with therefore more fluctuations. We also note that the short-time limit errors, already attributed in the main text to tracking errors, are present in every case. These errors draw a precision limit on $\epsilon$ which is due to the reduced statistics in the short-time limit, leading to an increased error term.

The ergodic estimator $\epsilon$ can be represented as a function of the lag $\Delta$ as in the main text, but can also be represented as a function of the time $\mathcal{T}$ as done in Ref. [16]. The expected simple dependence $\epsilon(\mathcal{T}) \sim \mathcal{T}^{-1}$ can clearly be seen in Fig. 11 .

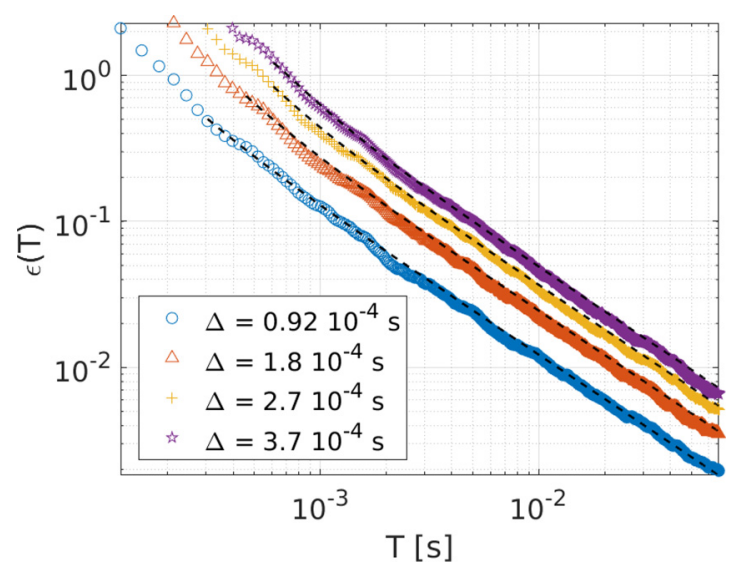

FIG. 11. Experimental ergodic estimator $\epsilon$ as a function of the total time $\mathcal{T}$ for different fixed values of the time lag $\Delta$. 


\section{APPENDIX G: ANALYTICAL EXPRESSION OF THE \\ ERGODIC ESTIMATOR FOR THE \\ ORNSTEIN-UHLENBECK PROCESS \\ (HARMONIC POTENTIAL)}

Under the condition of stationarity, the position correlation function depends only on the time lag $\Delta$, with

$$
C_{x}(\Delta)=\langle x(\Delta+t) x(t)\rangle=\frac{2 k_{B} T}{\kappa} e^{-\frac{\kappa}{\gamma} \Delta} .
$$

We note the definition of the ergodic estimator $\epsilon$ [15],

$$
\epsilon(\Delta)=\frac{\sigma^{2}\left(\overline{\delta x_{i}^{2}(\Delta)}\right)}{\left\langle\overline{\left.\delta x_{i}^{2}(\Delta)\right)}\right\rangle^{2}}
$$

where $\sigma^{2}\left(\overline{\delta x_{i}^{2}(\Delta)}\right)$ stands for the variance of a single trajectory time-averaged MSD,

$$
\overline{\delta x_{i}^{2}(\Delta)}=\frac{1}{\mathcal{T}-\Delta} \int_{0}^{\mathcal{T}-\Delta}\left[x_{i}\left(t^{\prime}+\Delta\right)-x_{i}\left(t^{\prime}\right)\right]^{2} d t^{\prime}
$$

and $\left\langle\overline{\left.\delta x_{i}^{2}(\Delta)\right)}\right\rangle$ stands for the mean of time-averaged MSD taken over the available ensemble $\{i\}$ of trajectories:

$$
\left\langle\overline{\delta x_{i}^{2}(\Delta)}\right\rangle=\frac{1}{\mathcal{T}-\Delta} \int_{0}^{\mathcal{T}-\Delta}\left\langle\left[x_{i}\left(t^{\prime}+\Delta\right)-x_{i}\left(t^{\prime}\right)\right]^{2}\right\rangle d t^{\prime} .
$$

Under the ergodic hypothesis, the time-ensemble averaged MSD is

$$
\left\langle\overline{\delta x_{i}^{2}(\Delta)}\right\rangle=\frac{2 k_{B} T}{\kappa}\left(1-e^{-\frac{\kappa}{\gamma} \Delta}\right)
$$

and the variance is defined as

$$
\sigma^{2}\left(\overline{\delta x_{i}^{2}(\Delta)}\right)=\left\langle{\overline{\delta x_{i}^{2}(\Delta)}}^{2}\right\rangle-\left\langle{\overline{\delta x_{i}^{2}(\Delta)}}^{2} .\right.
$$

The first term can be written as

$$
\begin{aligned}
\left\langle{\overline{\delta x_{i}^{2}(\Delta)}}^{2}\right\rangle= & \frac{1}{(\mathcal{T}-\Delta)^{2}} \int_{0}^{\mathcal{T}-\Delta} d t_{1} \int_{0}^{\mathcal{T}-\Delta} d t_{2} \\
& \times\left\langle\left(x\left(t_{1}+\Delta\right)-x\left(t_{1}\right)\right)^{2}\left(x\left(t_{2}+\Delta\right)-x\left(t_{2}\right)\right)^{2}\right\rangle,
\end{aligned}
$$

for which the Wick's relation yields four terms:

$$
\begin{aligned}
\left\langle x\left(t_{1}\right) x\left(t_{2}\right) x\left(t_{3}\right) x\left(t_{4}\right)\right\rangle= & \left\langle x\left(t_{1}\right) x\left(t_{2}\right)\right\rangle\left\langle x\left(t_{3}\right) x\left(t_{4}\right)\right\rangle \\
& +\left\langle x\left(t_{1}\right) x\left(t_{3}\right)\right\rangle\left\langle x\left(t_{2}\right) x\left(t_{4}\right)\right\rangle \\
& +\left\langle x\left(t_{1}\right) x\left(t_{4}\right)\right\rangle\left\langle x\left(t_{2}\right) x\left(t_{3}\right)\right\rangle .
\end{aligned}
$$

The integrand in Eq. (G7) then becomes

$$
\begin{aligned}
& \left\langle\left(x\left(t_{1}+\Delta\right)-x\left(t_{1}\right)\right)^{2}\left(x\left(t_{2}+\Delta\right)-x\left(t_{2}\right)\right)^{2}\right\rangle \\
& =\left[\left\langle\left(x\left(t_{1}+\Delta\right)-x\left(t_{1}\right)\right)^{2}\right\rangle\left\langle\left(x\left(t_{2}+\Delta\right)-x\left(t_{2}\right)\right)^{2}\right\rangle\right. \\
& \left.\quad+2\left\langle\left(x\left(t_{1}+\Delta\right)-x\left(t_{1}\right)\right)\left(x\left(t_{2}+\Delta\right)-x\left(t_{2}\right)\right)\right\rangle^{2}\right] .
\end{aligned}
$$

With the first term on the left-hand side of Eq. (G9) identified as the square of the time-ensemble averaged $\operatorname{MSD}\left\langle\overline{\delta x_{i}^{2}(\Delta)}\right\rangle^{2}$, the variance of the time-averaged MSD can finally be written as

$\sigma^{2}\left(\overline{\delta x_{i}^{2}(\Delta)}\right)=\frac{2}{(\mathcal{T}-\Delta)^{2}} \int_{0}^{\mathcal{T}-\Delta} d t_{1} \int_{0}^{\mathcal{T}-\Delta} d t_{2}$

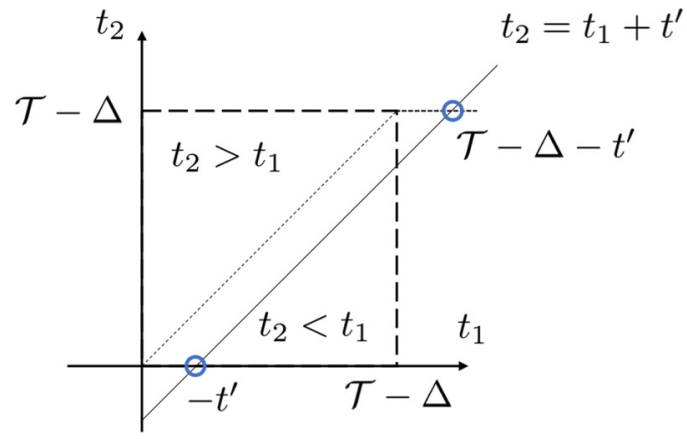

FIG. 12. Integration surface for Eq. (G11) on which the two sectors $\left[t_{2}>t_{1}\right]$ and $\left[t_{2}<t_{1}\right]$ are distinguished. This defines the appropriate change of variables $\left(t_{1}, t_{2}\right) \leftrightarrow\left(t_{1}, t^{\prime}\right)$, with the line $t_{2}=$ $t_{1}+t^{\prime}$ crossing the $t_{2}=0$ axis at $-t^{\prime}$ and the $t_{2}=\mathcal{T}-\Delta$ axis at $\mathcal{T}-\Delta-t^{\prime}$.

$$
\begin{aligned}
& \times\left\langle\left(x\left(t_{1}+\Delta\right)-x\left(t_{1}\right)\right)\left(x\left(t_{2}+\Delta\right)-x\left(t_{2}\right)\right)\right\rangle^{2} \\
= & \frac{2 k_{B}^{2} T^{2}}{(\mathcal{T}-\Delta)^{2} \kappa^{2}} \int_{0}^{\mathcal{T}-\Delta} d t_{1} \int_{0}^{\mathcal{T}-\Delta} d t_{2} \\
& \times\left(2 e^{-\frac{\kappa}{\gamma}\left|t_{1}-t_{2}\right|}-e^{-\frac{\kappa}{\gamma}\left|t_{1}-t_{2}+\Delta\right|}-e^{-\frac{\kappa}{\gamma}\left|t_{2}-t_{1}+\Delta\right|}\right)^{2},
\end{aligned}
$$

using Eq. (G1).

The integral is calculated through a standard change of variables $t_{1}=t_{1}, t^{\prime}=t_{2}-t_{1}$ described in Fig. 12 and is possible since the integrand only depends on the $\left|t_{1}-t_{2}\right|$ difference. One can formally write

$$
\sigma^{2}\left(\overline{\delta x_{i}^{2}(\Delta)}\right)=\frac{2 k_{B}^{2} T^{2}}{(\mathcal{T}-\Delta)^{2} \kappa^{2}} \int_{0}^{\mathcal{T}-\Delta} d t_{1} \int_{0}^{\mathcal{T}-\Delta} d t_{2} \alpha^{2}\left(t^{\prime}\right)
$$

with $t^{\prime}$ varying from negative to positive values in the $\left(t_{1}, t_{2}\right)$ plane. For the $t^{\prime}>0$ sector,

$$
\begin{aligned}
& \int_{0}^{\mathcal{T}-\Delta} d t^{\prime} \int_{0}^{\mathcal{T}-\Delta-t^{\prime}} d t_{1} \alpha^{2}\left(t^{\prime}\right) \\
& \quad=\int_{0}^{\mathcal{T}-\Delta} d t^{\prime}\left(\mathcal{T}-\Delta-t^{\prime}\right) \alpha^{2}\left(t^{\prime}\right),
\end{aligned}
$$

and for the $t^{\prime}<0$ sector:

$$
\begin{aligned}
& \int_{-(\mathcal{T}-\Delta)}^{0} d t^{\prime} \int_{-t^{\prime}}^{\mathcal{T}-\Delta} d t_{1} \alpha^{2}\left(t^{\prime}\right) \\
& \quad=\int_{-(\mathcal{T}-\Delta)}^{0} d t^{\prime}\left(\mathcal{T}-\Delta+t^{\prime}\right) \alpha^{2}\left(t^{\prime}\right) .
\end{aligned}
$$

By combining the two two sectors, one gets

$$
\begin{aligned}
& \int_{-(\mathcal{T}-\Delta)}^{\mathcal{T}-\Delta)} d t^{\prime}\left(\mathcal{T}-\Delta-\left|t^{\prime}\right|\right) \alpha^{2}\left(t^{\prime}\right) \\
& =2 \int_{0}^{\mathcal{T}-\Delta} d t^{\prime}\left(\mathcal{T}-\Delta-\left|t^{\prime}\right|\right) \alpha^{2}\left(t^{\prime}\right) \\
& =2 \int_{0}^{\mathcal{T}-\Delta} d t^{\prime}\left(\mathcal{T}-\Delta-t^{\prime}\right) \alpha^{2}\left(t^{\prime}\right)
\end{aligned}
$$


leading to express the ergodic estimator $\epsilon$ as

$$
\begin{aligned}
\epsilon(\Delta)= & \frac{4 k_{B}^{2} T^{2}}{\kappa^{2}(\mathcal{T}-\Delta)^{2}\left\langle\overline{\delta x_{i}^{2}(\Delta)}\right\rangle^{2}} \\
& \times \int_{0}^{\mathcal{T}-\Delta} d t^{\prime}\left(\mathcal{T}-\Delta-t^{\prime}\right)\left(2 e^{-\frac{k t^{\prime}}{\gamma}}-e^{-\frac{\kappa}{\gamma}\left(t^{\prime}+\Delta\right)}\right. \\
& \left.-e^{-\frac{\kappa}{\gamma}\left|\Delta-t^{\prime}\right|}\right)^{2}
\end{aligned}
$$

To simplify the notations, we define $K=\frac{k_{B} T}{\kappa}$ and write the time-ensemble averaged MSD as $\left\langle\overline{\delta x_{i}^{2}(\Delta)}\right\rangle=2 K\left(1-e^{-\frac{\kappa}{\gamma} \Delta}\right)$.
The ergodic estimator is then written as $\epsilon(\Delta)=I / 4 K^{2}(1-$ $\left.e^{-\frac{\kappa}{\gamma} \Delta}\right)^{2}$ where the variance of the MSD $I$ is calculated as

$$
\begin{array}{r}
I=\frac{4 K^{2}}{(\mathcal{T}-\Delta)^{2}} \int_{0}^{\mathcal{T}-\Delta} d t^{\prime}\left(\mathcal{T}-\Delta-t^{\prime}\right) \\
\left(2 e^{-\frac{\kappa t^{\prime}}{\gamma}}-e^{-\frac{\kappa}{\gamma}\left(t^{\prime}+\Delta\right)}-e^{-\frac{\kappa}{\gamma}\left|\Delta-t^{\prime}\right|}\right)^{2},
\end{array}
$$

split in three terms depending on the sign of the absolute value:

$$
\begin{aligned}
= & \frac{4 K^{2}}{(\mathcal{T}-\Delta)^{2}} \mathcal{T} \int_{0}^{\Delta}\left(2 e^{-\frac{\kappa t^{\prime}}{\gamma}}-e^{-\frac{\kappa}{\gamma}\left(t^{\prime}+\Delta\right)}-e^{-\frac{\kappa}{\gamma}\left(\Delta-t^{\prime}\right)}\right)^{2} d t^{\prime} \\
& -\frac{4 K^{2}}{(\mathcal{T}-\Delta)^{2}} \int_{0}^{\Delta}\left(t^{\prime}+\Delta\right)\left(2 e^{-\frac{\kappa t^{\prime}}{\gamma}}-e^{-\frac{\kappa}{\gamma}\left(t^{\prime}+\Delta\right)}-e^{-\frac{\kappa}{\gamma}\left(\Delta-t^{\prime}\right)}\right)^{2} d t^{\prime} \\
& +\frac{4 K^{2}}{(\mathcal{T}-\Delta)^{2}} \int_{\Delta}^{\mathcal{T}-\Delta}\left(\mathcal{T}-\Delta-t^{\prime}\right)\left(2 e^{-\frac{\kappa t^{\prime}}{\gamma}}-e^{-\frac{\kappa}{\gamma}\left(t^{\prime}+\Delta\right)}-e^{\frac{\kappa}{\gamma}\left(\Delta-t^{\prime}\right)}\right)^{2} d t^{\prime} \\
= & V_{1}+V_{2}+V_{3} .
\end{aligned}
$$

Each term is calculated as

$$
\begin{aligned}
V_{1}= & \frac{4 K^{2}}{(\mathcal{T}-\Delta)^{2}} \cdot \frac{\gamma \mathcal{T}}{2 \kappa}\left[5+\frac{4 \kappa}{\gamma}\left(e^{-\frac{2 \kappa}{\gamma} \Delta}-2 e^{-\frac{\kappa}{\gamma} \Delta}\right)-4 e^{-\frac{2 \kappa}{\gamma} \Delta}+4 e^{-\frac{3 \kappa}{\gamma} \Delta}-4 e^{-\frac{\kappa}{\gamma} \Delta}-e^{-\frac{4 \kappa}{\gamma} \Delta}\right] \\
V_{2}= & \frac{K^{2}}{(\mathcal{T}-\Delta)^{2}} \cdot \frac{\gamma^{2}}{\kappa^{2}}\left[\left(4 \frac{\kappa}{\gamma} \Delta-1\right)+12 \frac{\kappa^{2}}{\gamma^{2}} \Delta^{2} e^{-2 \frac{\kappa}{\gamma} \Delta}\left(1-2 e^{\frac{\kappa}{\gamma} \Delta}\right)+\frac{2 \kappa}{\gamma} e^{-\frac{4 \kappa}{\gamma} \Delta} \Delta\left(-4 e^{2 \frac{\kappa}{\gamma} \Delta}+4 e^{\frac{\kappa}{\gamma} \Delta}-1\right)\right. \\
& \left.+\left(\frac{2 \kappa}{\gamma} \Delta+1\right)\left(-4 e^{-2 \frac{\kappa}{\gamma} \Delta}+4 e^{-3 \frac{\kappa}{\gamma} \Delta}-4 e^{-\frac{\kappa}{\gamma} \Delta}-e^{-4 \frac{\kappa}{\gamma} \Delta}+4\right)+2 e^{-2 \frac{\kappa}{\gamma} \Delta}\right] \\
V_{3}= & \frac{16 K^{2}}{(\mathcal{T}-\Delta)^{2}}\left(\cosh \left(\frac{\kappa}{\gamma} \Delta\right)-1\right)^{2}\left\{\frac{\gamma}{2 \kappa}(\mathcal{T}-\Delta)\left(e^{-2 \frac{\kappa}{\gamma} \Delta}-e^{-2 \frac{\kappa}{\gamma}(\mathcal{T}-\Delta)}\right)+\frac{\gamma^{2}}{4 \kappa^{2}}\left[\left(\frac{2 \kappa}{\gamma}(\mathcal{T}-\Delta)+1\right) e^{-2 \frac{\kappa}{\gamma}(\mathcal{T}-\Delta)}\right.\right. \\
& \left.\left.-\left(\frac{2 \kappa}{\gamma} \Delta+1\right) e^{-2 \frac{\kappa}{\gamma} \Delta}\right]\right\}
\end{aligned}
$$

whose analytical expression is drawn as the theory curve in Fig. 6 in the main text.

\section{APPENDIX H: TRACKING ERROR ANALYSIS}

\section{Tracking error on position}

In all our experiments, the trajectories are recorded by a photodiode and the positions are interpreted from the photodiode signal. The errors on the localization of the particle in our experiments originate from multiple noise sources dominated by the laser fluctuation and the diode electronic noise. A white noise can be a good starting approximation to estimate and describe the localization error. Therefore, each measured position $x_{i}\left(t_{k}\right)$ for a trajectory $i$ at time $t_{k}$ can be related to the real position $x_{i}^{0}\left(t_{k}\right)$ as $[10,32]$

$$
x_{i}\left(t_{k}\right)=x_{i}^{0}\left(t_{k}\right)+\mu_{i}\left(t_{k}\right)
$$

where $\mu_{i}\left(t_{k}\right)$ is a random uncorrelated tracking error with $\left\langle\mu_{i}\left(t_{k}\right)\right\rangle=0$ and $\left\langle\mu_{i}\left(t_{k}\right) \mu_{j}\left(t_{l}\right)\right\rangle=\delta_{i j} \delta_{k l} \sigma_{0}^{2}$.

\section{Tracking error on time-ensemble averaged MSD}

We now propagate the position tracking error described by Eq. (H1) into the measured MSD. We write

$$
\begin{aligned}
& \left\langle\overline{\left(x_{i}(t+\Delta)-x_{i}(t)\right)^{2}}\right\rangle \\
& =\left\langle\overline{\left(x_{i}^{0}(t+\Delta)+\mu_{i}(t+\Delta)-x_{i}^{0}(t)-\mu_{i}(t+\Delta)\right)^{2}}\right\rangle \\
& =\left\langle\overline{\left[\left(x_{i}^{0}(t+\Delta)-x_{i}^{0}(t)\right)+\left(\mu_{i}(t+\Delta)-\mu_{i}(t)\right)\right]^{2}}\right\rangle \\
& \left.=\overline{\left(x_{i}^{0}(t+\Delta)-x_{i}^{0}(t)\right)^{2}}\right\rangle+\left\langle\overline{\left(\mu_{i}(t+\Delta)-\mu_{i}(t)\right)^{2}}\right\rangle \\
& =\left\langle\overline{\left(x_{i}^{0}(t+\Delta)-x_{i}^{0}(t)\right)^{2}}\right\rangle+2 \sigma_{0}^{2},
\end{aligned}
$$

showing how the measured MSD can be related to the theoretical one as

$$
\left\langle\overline{\delta x^{2}(\Delta)}\right\rangle_{\exp }=\left\langle\overline{\delta x^{2}(\Delta)}\right\rangle_{\mathrm{th}}+2 \sigma_{0}^{2} .
$$



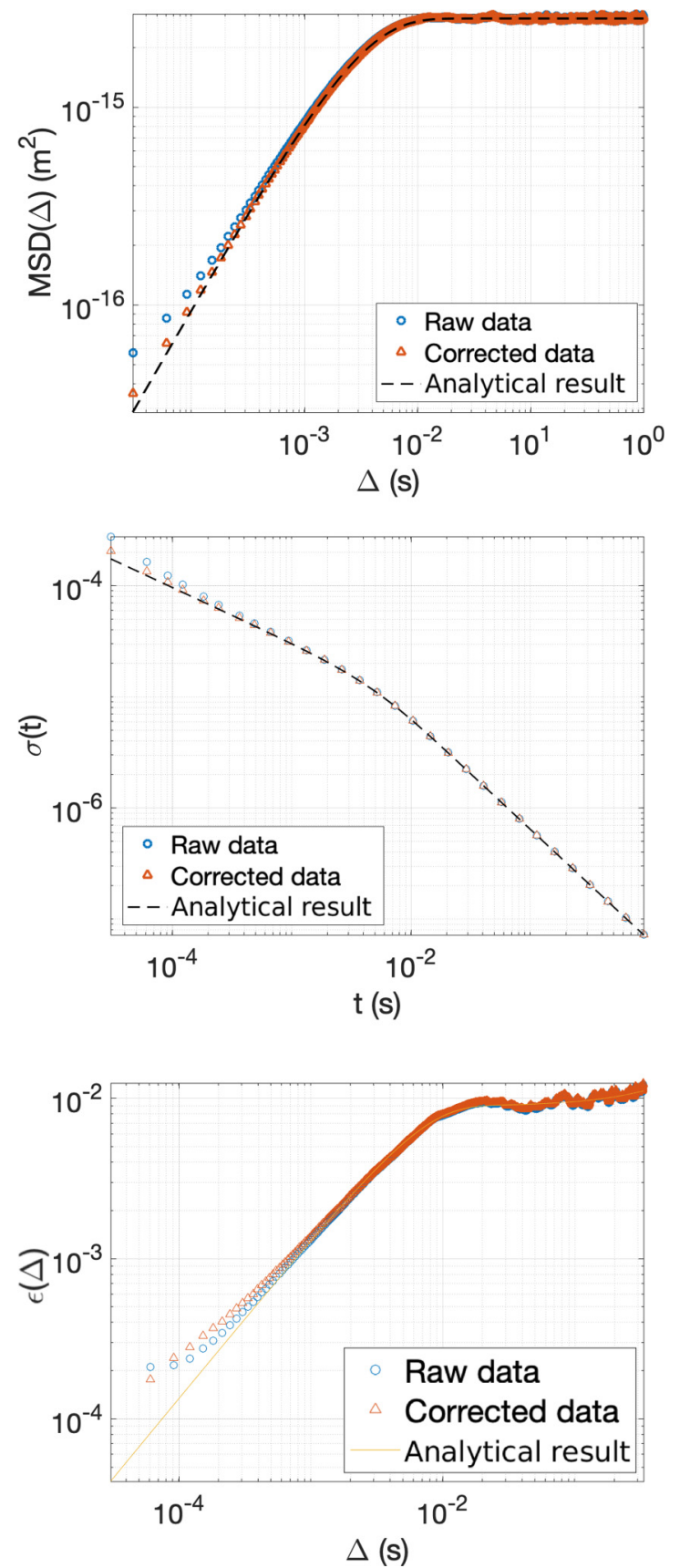

FIG. 13. Raw experimental and corrected data (top) for the MSD (middle) for the Allan variance and (bottom) for the ergodic estimator. We see the correction mostly for short time lags. The correction works well for the MSD and Allan variance, but a deviation remains on the ergodic estimator. This difference could actually point to a slight deviation of the localization noise from the white Gaussian noise we have assumed in our modeling of the localization error.

Since $\sigma_{0}^{2}>0$, the MSD is always overdetermined experimentally, in agreement with our observations; in the log-log representation of Fig. 5, this error can mainly be seen at short time lags.

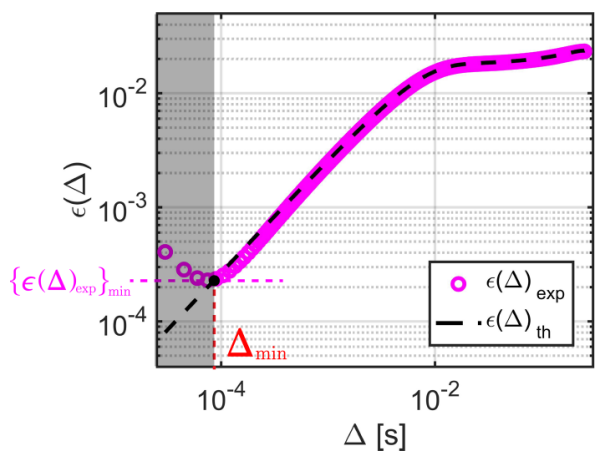

FIG. 14. Evolution of the experimental estimator $\epsilon(\Delta)_{\exp }$ described by Eq. (H9) and plotted using a fixed MSD error variance $\sigma^{2}\left(\varepsilon_{i}\right)=9.63 \times 10^{-37} \mathrm{~m}^{4}$. This gives a minimal value $\left\{\epsilon(\Delta)_{\exp }\right\}_{\min }$ that, compared to the analytical curve $\left(\epsilon(\Delta)_{\text {th }}\right)$, shows the exclusion zone limited by $\Delta_{\min }$.

\section{Tracking error on Allan variance}

From the definition of Allan variance, we can also relate the experimental Allan variance that includes the tracking errors to the theoretical Allan variance with

$$
\begin{aligned}
\sigma_{\exp }^{2}(\Delta)= & \frac{1}{2 \Delta^{2}}\left\langle(x((n+2) \Delta)-2 x((n+1) \Delta)+x(\Delta))^{2}\right\rangle \\
= & \frac{1}{2 \Delta^{2}}\left\langle\left( x^{0}((n+2) \Delta)-2 x^{0}((n+1) \Delta)+x^{0}(\Delta)\right.\right. \\
& \left.\left.+\mu_{i}-2 \mu_{j}+\mu_{k}\right)^{2}\right\rangle \\
= & \sigma_{\mathrm{th}}^{2}(\Delta)+\frac{1}{2 \Delta^{2}}\left\langle\left(\mu_{i}-2 \mu_{j}+\mu_{k}\right)^{2}\right\rangle \\
= & \sigma_{\mathrm{th}}^{2}(\Delta)+\frac{3 \sigma_{0}^{2}}{\Delta^{2}} .
\end{aligned}
$$

The difference $3 \sigma_{0}^{2} / \Delta^{2}$ between experimental and theoretical Allan variances is always positive and decays with $\Delta^{2}$, again a feature perfectly consistent with our observations--see Fig. 6 in the main text.

\section{Tracking error on the ergodic estimator}

To account for the error on the ergodic estimator $\epsilon(\Delta)$, we first consider Eq. (H3) for the MSD error analysis. For the single trajectory time-averaged MSD, one has

$$
{\overline{\delta x_{i}^{2}(\Delta)}}_{\exp }={\overline{\delta x_{i}^{2}(\Delta)_{\mathrm{th}}}}+\varepsilon_{i},
$$

where $\varepsilon_{i}$ is a random constant with $\left\langle\varepsilon_{i}\right\rangle=2 \sigma_{0}^{2}$. The experimental ergodic estimator can thus be written as

$$
\epsilon(\Delta)_{\exp }=\frac{\left\langle\left({\overline{\delta x_{i}^{2}(\Delta)_{\exp }}}\right)^{2}\right\rangle}{\left\langle{\overline{\delta x_{i}^{2}(\Delta)_{\exp }}}^{2}\right.}-1=\frac{\left\langle\left({\overline{\delta x_{i}^{2}(\Delta)_{\mathrm{th}}}}+\varepsilon_{i}\right)^{2}\right\rangle}{\left\langle{\overline{\delta x_{i}^{2}(\Delta)}}_{\exp }\right\rangle^{2}}-1 .
$$

We define the ratio

$$
\phi(\Delta)=\frac{\left\langle{\overline{\delta x_{i}^{2}(\Delta)_{\mathrm{th}}}}\right\rangle}{\left\langle\overline{\delta x_{i}^{2}(\Delta)_{\exp }}\right\rangle}
$$

as the ratio between the theoretical and experimental MSD variance value. With this ratio, the experimental 
ergodic estimator $\epsilon(\Delta)$ can be written as

$$
\begin{aligned}
\epsilon(\Delta)_{\exp }= & \phi^{2}(\Delta) \epsilon(\Delta)_{\mathrm{th}} \\
& +\phi^{2}(\Delta)\left(\frac{2\left\langle\varepsilon_{i} \overline{\delta x_{i}^{2}(\Delta)_{\mathrm{th}}}\right\rangle+\left\langle\varepsilon_{i}^{2}\right\rangle}{\left.\overline{\left\langle\overline{x i}_{i}^{2}(\Delta)_{\mathrm{th}}\right.}\right\rangle^{2}}+1\right)-1 .
\end{aligned}
$$

Assuming that the error $\varepsilon_{i}$ is uncorrelated with the single trajectory time ensemble MSD, $\left\langle\varepsilon_{i} \overline{\delta x_{i}^{2}(\Delta)_{\text {th }}}\right\rangle=\left\langle\varepsilon_{i}\right\rangle\left\langle\overline{\delta x_{i}^{2}(\Delta)_{\text {th }}}\right\rangle$. Taking this into account additionally leads to $\sigma^{2}\left(\varepsilon_{i}\right)=\left\langle\varepsilon_{i}^{2}\right\rangle-$ $\left\langle\varepsilon_{i}\right\rangle^{2}$ and therefore to

$$
\epsilon(\Delta)_{\exp }=\phi^{2}(\Delta)\left[\epsilon(\Delta)_{\mathrm{th}}+\frac{\sigma^{2}\left(\varepsilon_{i}\right)}{\left\langle\left.\overline{\delta x_{i}^{2}(\Delta)_{\mathrm{th}}}\right|^{2}\right.}\right] .
$$

Using Eq. (G5), the ratio

$$
\phi(\Delta)=\frac{1}{1+\frac{\kappa \sigma_{0}^{2}}{k_{B} T\left(1-e^{-\kappa \Delta / \gamma}\right)}}
$$

can be estimated once the value of the localization error $\sigma_{0}^{2}$ is known. As for the variance of $\varepsilon_{i}$, Eq. (H5) gives

$$
\sigma^{2}\left(\varepsilon_{i}\right)=\sigma^{2}\left({\overline{\delta x_{i}^{2}(\Delta)}}_{\exp }\right)-\sigma^{2}\left({\overline{\delta x_{i}^{2}(\Delta)}}_{\mathrm{th}}\right) .
$$

Since $\sigma^{2}\left({\overline{\delta x_{i}^{2}(\Delta)}}_{\text {th }}\right)$ goes to zero when $\Delta \rightarrow 0$, one is left, at small $\Delta$, with $\sigma^{2}\left(\varepsilon_{i}\right) \sim \sigma^{2}\left({\overline{\delta x_{i}^{2}(\Delta)}}_{\exp }\right)$. Taking the experimental variance measured on the time-averaged MSD for the smallest time lag $\Delta$ is therefore a good estimation for $\sigma^{2}\left(\varepsilon_{i}\right)$. This analysis leads us to approach the real value of the tracking error on the estimator $\epsilon(\Delta)$ and this way explain the difference between the experimental data and the theoretical curve in Figs. 6 and 9.

However, it is seen in Fig. 13 that the correction of the estimator $\epsilon(\Delta)$ is limited. This is due to the fact that for such a correction, the estimation of $\sigma^{2}\left(\varepsilon_{i}\right)$ used is assumed to be constant, while it turns out to depend on $\Delta$. This dependence plays particularly when the number of sampling is limited. From its expression in Eq. (H9), the experimental estimator $\epsilon(\Delta)_{\exp }$ has a minimum that depends on $\sigma^{2}\left(\varepsilon_{i}\right)$. This minimal value stems from the localization errors but is difficult to measure experimentally. This difficulty is well seen from the experimental estimators $\epsilon(\Delta)$ evaluated from different subtrajectory samplings involving the different duration choices displayed in Fig. 10. There, deviations at small $\Delta$ for different numbers of subtrajectory samplings are clearly seen and yield signatures that look similar to ergodicity breaking. This similarity implies that when ergodicity is investigated by looking at the decay of the estimator $\epsilon(\Delta)$ as a function of $\Delta$, a lower limit in $\Delta$ must be chosen to ensure that the data are well above the experimental noise limit. This limit $\Delta_{\min }$ is set where, for a given estimated $\sigma^{2}\left(\varepsilon_{i}\right)$,

$$
\epsilon\left(\Delta_{\min }\right)_{\mathrm{th}}=\left\{\epsilon(\Delta)_{\exp }\right\}_{\min },
$$

as illustrated in Fig. 14. For a given trajectory length, the less the number of sampling, the larger $\Delta_{\min }$ is. Therefore, when the number of trajectories in an ensemble is limited, it is safer and better to evaluate the estimator $\epsilon(\Delta)$ as a function of both $\Delta$ and total trajectory length $\mathcal{T}$ at the same time.
[1] E. Lutz and S. Ciliberto, Phys. Today 68, 30 (2015).

[2] S. Ciliberto, Phys. Rev. X 7, 021051 (2017).

[3] I. A. Martinez, E. Roldán, L. Dinis, and R. Rica, Soft Matter 13, 22 (2017).

[4] J. Bechhoefer, S. Ciliberto, S. Pigolotti, and E. Roldán, J. Stat. Mech. (2020) 064001.

[5] P. Wu, R. Huang, C. Tischer, A. Jonas, and E.-L. Florin, Phys. Rev. Lett. 103, 108101 (2009).

[6] D. S. Ether, L. B. Pires, S. Umrath, D. Martinez, Y. Ayala, B. Pontes, G. R. de S. Araújo, S. Frases, G.-L. Ingold, F. S. S. Rosa, N. B. Viana, H. M. Nussenzveig, and P. A. M. Neto, Europhys. Lett. 112, 44001 (2015).

[7] F. Ricci, R. A. Rica, M. Spasenović, J. Gieseler, L. Rondin, L. Novotny, and R. Quidant, Nat. Commun. 8, 15141 (2017).

[8] L. Liu, S. Kheifets, V. Ginis, and F. Capasso, Phys. Rev. Lett. 116, 228001 (2016).

[9] G. Schnoering, Y. Rosales-Cabara, H. Wendehenne, A. Canaguier-Durand, and C. Genet, Phys. Rev. Appl. 11, 034023 (2019).

[10] M. Li, O. Sentissi, S. Azzini, G. Schnoering, A. CanaguierDurand, and C. Genet, Phys. Rev. A 100, 063816 (2019).

[11] V. Blickle and C. Bechinger, Nat. Phys. 8, 143 (2011).

[12] A. Berut, A. Arakelyan, A. Petrosyan, S. Ciliberto, R. Dillenschneider, and E. Lutz, Nature (London) 483, 7388 (2012).
[13] I. A. Martínez, A. Petrosyan, D. Guéry-Odelin, E. Trizac, and S. Ciliberto, Nat. Phys. 12, 843 (2016).

[14] Y. Rosales-Cabara, G. Manfredi, G. Schnoering, P.-A. Hervieux, L. Mertz, and C. Genet, Phys. Rev. Res. 2, 012012(R) (2020).

[15] R. Metzler, J.-H. Jeon, A. G. Cherstvy, and E. Barkai, Phys. Chem. Chem. Phys. 16, 24128 (2014).

[16] A. G. Cherstvy and R. Metzler, J. Stat. Mech. (2015) P05010.

[17] A. G. Cherstvy, S. Thapa, Y. Mardoukhi, A. V. Chechkin, and R. Metzler, Phys. Rev. E 98, 022134 (2018).

[18] G. Uhlenbeck and L. Ornstein, Phys. Rev. 36, 823 (1930).

[19] C. Gardiner, Stochastic Methods: A Handbook for the Natural and Social Sciences, 4th ed. (Springer-Verlag, Berlin, 2009), Vol. 13 .

[20] K. Neuman and S. Block, Rev. Sci. Instrum. 75, 2787 (2004).

[21] G. Volpe and G. Volpe, Am. J. Phys. 81, 224 (2013).

[22] P. E. Kloeden and E. Platen, Numerical Solutions of Stochastic Differential Equations, 1st ed. (Springer-Verlag, Berlin, 1992), Vol. 23.

[23] D. Higham, SIAM Rev. 43, 525 (2001).

[24] E. Vanden-Eijnden and G. Ciccotti, Chem. Phys. Lett. 429, 310 (2006). 
[25] F. Czerwinski, A. C. Richardson, and L. B. Oddershede, Opt. Express 17, 13255 (2009).

[26] B. M. Lansdorp and O. A. Saleh, Rev. Sci. Instrum. 83, 025115 (2012).

[27] D. W. Allan, Proc. IEEE 54, 221 (1966).

[28] J. A. Barnes, A. R. Chi, L. S. Cutler, D. J. Healey, D. B. Leeson, T. E. McGunigal, J. A. Mullen, W. L. Smith, R. L. Sydnor, R. F. Vessot, and G. M. R. Winckler, IEEE Trans. Instrum. Meas. 20, 105 (1971).
[29] C. Bechinger, R. Di Leonardo, H. Löwen, C. Reichhardt, G. Volpe, and G. Volpe, Rev. Mod. Phys. 88, 045006 (2016).

[30] A. Ghosh and D. Chakraborty, J. Chem. Phys. 152, 174901 (2020).

[31] G. Schnoering, L. V. Poulikakos, Y. Rosales-Cabara, A. Canaguier-Durand, D. J. Norris, and C. Genet, Phys. Rev. Lett. 121, 023902 (2018).

[32] X. Michalet and A. J. Berglund, Phys. Rev. E 85, 061916 (2012). 